\title{
Optimizing 802.11 Wireless Mesh Networks Based on Physical Carrier Sensing
}

\author{
Hui Ma, Student Member, IEEE, Rajiv Vijayakumar, Sumit Roy, Fellow, IEEE, and Jing Zhu, Member, IEEE
}

\begin{abstract}
Multi-hop ad hoc networks suffer from the "hidden" and "exposed" node problems which diminish aggregate network throughput. While there are various approaches to mitigating these problems, in this work we focus exclusively on the role of physical carrier sensing (PCS). Specifically, tuning the PCS threshold leads to a trade-off between the hidden and exposed cases; reducing one typically increases the other, implying the existence of an optimal PCS threshold setting maximizes the aggregate network throughput. The contributions of this work are two-fold:

i. We develop an analytical model to determine the optimal PCS threshold for a homogeneous network with constant link distances and show that setting the carrier sensing range close to the interference range is a robust close-to-optimal setting for network optimization in many scenarios. As an extension to more pragmatic network topologies with non-uniform link distances, a rate-to-link allocation scheme is proposed based on rendering the interference range equal for all links that allows a single carrier sense range to be used for the whole network.

ii. The above suggests the need for on-line adaptation of tunable PCS threshold in general. The proposed algorithm is based on the key concept of loss differentiation (LD), which disambiguates the cause of packet loss event due to link layer interference (hidden terminals) and collisions respectively. Extensive simulation results show that the proposed PCS adaptations make the PCS threshold converge to its optimal value and thus outperform schemes without PCS adaptation.
\end{abstract}

Index Terms-80211 WLAN, hidden and exposed terminals, loss differentiation, multi-rate, OPNET simulation, physical carrier sensing, throughput optimization.

\section{INTRODUCTION}

$\mathbf{P}$ HYSICAL carrier sensing (PCS) is one of the two main interference mitigation mechanisms used in the PHY/MAC layers of 802.11 WLANs, the other being virtual carrier sensing (VCS) via the use of RTS/CTS signaling. PCS is generically termed as Clear Channel Assessment (CCA) and involves estimating channel status surrounding a node. In PCS, a node that intends to transmit assesses channel state, for example, by comparing the measured on-air received energy in a channel against

Manuscript received August 15, 2007; revised April 11, 2008 and April 11, 2008; approved by IEEE/ACM Transactions on Networking Editor A. Kumar. This work was supported in part by Intel Corporation.

H. Ma and S. Roy are with the Department of Electrical Engineering, University of Washington, Seattle, WA 98195 USA (e-mail: mahui@u.washington. edu).

R. Vijayakumar is with Qualcomm, Inc., San Diego, CA 92121 USA.

$\mathrm{J}$. Zhu is with the Communications Technology Lab, Intel Corporation, Hillsboro, OR 97124 USA.

Color versions of one or more of the figures in this paper are available online at http://ieeexplore.iee.org.

Digital Object Identifier 10.1109/TNET.2008.2009443 a predefined PCS threshold (i.e., energy detection). ${ }^{1}$ A node initiates channel access only if the detected value is below the threshold, indicating that the channel is free of significant ongoing transmissions. In this work, we will consider the case of a homogeneous (identical nodes, uniform spatial density) network whereby all nodes use the same transmit power and PCS threshold. The PCS threshold thus effectively defines a carrier sensing range that denotes an area wherein a secondary transmitter is prevented from contending for access so as to not disrupt the desired transmission.

However (as is well known), the sensed channel state at the transmitter's location is not always an accurate predictor of the channel state seen by the receiver, leading to the well known hidden and exposed terminal problems, both of which degrade aggregate throughput. The hidden terminal case arises when a transmitted packet is not successfully received at the receiver due to interference from a secondary transmitter whose presence was not detected by the reference transmitter. This occurs when the reference transmitter's PCS threshold is too high to detect the presence of the interferer. Conversely, the exposed terminal case occurs when a transmitter refrains from transmitting even though the interference at the intended receiver is low enough to have allowed for a successful transmission. In this case, the reference transmitter's PCS threshold is too low causing it to (needlessly) defer to secondary transmissions which do not cause undue interference. The hidden terminal case lowers throughput due to the loss of transmitted packets whereas the exposed terminal case lowers throughput by unnecessarily suppressing transmissions that could have occurred successfully. ${ }^{2}$

The preferred approach to collision avoidance in the 802.11 literature has leaned towards use of RTS/CTS, i.e., VCS whereby the sender-destination pair engages in a handshake to reserve the channel prior to actual data transmission. All nodes within a transmission range $R_{t r}$ of both the sender and the receiver defer transmission by this mechanism for the duration of the subsequent DATA and ACK; however it is easy to see that whenever the interference range $R_{I}>R_{t r}$, a secondary source within the interference range but outside the transmission range of the destination receiver will cause loss of desired packet even if RTS/CTS were employed. This point was investigated in depth in [3] where it was shown that VCS

\footnotetext{
${ }^{1}$ In this work, energy detection based PCS is assumed because a) it is a universal (it will always be implemented in some form), low-cost (in terms of power dissipation) approach and b) it is easier to model analytically. Study of other modes of PCS, i.e., preamble detection (PD) or a combination of PD and ED, will be deferred to future work.

${ }^{2}$ In this work, we exclusively focus on aggregate network throughput as our performance metric; we defer considerations of important topics such as per node throughput fairness to later work.
} 
is optimal when $R_{t r}=R_{I}$, i.e., the transmission range equals the interference range. However, we underscore the difficulty of achieving this in practice due to the fact that $R_{I}$ is implicitly a function of $R_{t r}$ and cannot be independently tuned to achieve the desired equality. Further note that in the 802.11 standard, the use of RTS/CTS has been provided as an option for longer payloads only, and it is not recommended for short packets due to the additional overhead of the RTS/CTS exchange. In fact, RTS/CTS is triggered based on packet size and is not, generally speaking, under user control and thus cannot be relied on for network optimization. We refer the reader to [5] which shows several examples where the use of RTS/CTS actually leads to lower throughput than if it were disabled.

For all these reasons, we have argued in prior work [1] for a deeper investigation of PCS as a pragmatic preferred alternative to VCS for optimizing aggregate network throughput. The fundamental premise is that PCS allows more fine grained control and tuning of network performance than is possible through VCS for two reasons: i. PCS threshold is a parameter independent of transmit/interference range, and ii. PCS is mandatory in the 802.11 standard. Several current 802.11 WLAN hardware/firmware implementations support one or more parameters for PCS control; some of these are available at run-time for user definition via open source Linux drivers such as IPW2200 [6] for Intel's Pro/Wireless chipsets.

In this paper, we first develop a simple yet effective model for incorporating the impact of hidden and exposed terminals in a spatially homogeneous mesh network that yields an optimization problem in terms of the PCS threshold. We show that choosing the PCS threshold such that the CS range equals the interference range is a robust close-to-optimal setting. This next motivates a rate-to-link allocation scheme for networks with random link distances based rendering the interference range equal for all links; this in turn allows a single carrier sense range to be used for improving network throughput.

As is apparent from the above, tuning of mesh (ad hoc) networks require practical run-time algorithms for PCS adaptation since perfect knowledge of parameters assumed in the analytical model are generally unavailable. Real-time measurement of link state as contained in the packet error rate (PER) is the driver for such adaptation. However, differentiating the causes of packet errors-between losses due to asynchronous interference (hidden terminals) and synchronous collision-is important for appropriate network tuning. In dense networks, interference from hidden terminals likely dominate; nonetheless, any adaptation scheme that does not consider collisions can lead to lower-than-optimal aggregate throughput or even divergence of the algorithm. Therefore, our proposed algorithm is based on the key concept of loss differentiation, which disambiguates the above causes in packet errors respectively. Extensive simulation results show that the proposed PCS adaptations (along with the rate-to-link allocation) make the PCS threshold converge to its optimal value and thus outperform schemes without PCS adaptation.

The rest of this paper is organized as follows. We first summarize related work in Section II and introduce the link layer model in Section III. In Section IV, we develop an analytical model to determine the optimal carrier sensing range and then verify it with simulations. Motivated by the analytical model, a link adaptation algorithm is proposed in Section V. Next, the PCS adaptation algorithm based on loss differentiation is proposed and then evaluated by simulations in Section VI. Finally, we conclude the paper in Section VII.

\section{RELATED WORK}

Recent efforts dedicated to enhancing spatial reuse by PCS threshold adaptation include Vasan et al. [9] that proposed the ECHOS algorithm for an infrastructure mode WLAN whereby each AP selects the PCS threshold for all clients in the cell. On the other hand, Zhu et al. [1] derived the optimal PCS threshold for a regular mesh network that maximizes the aggregate one-hop throughput given a minimum required SNR; an adaptive PCS threshold algorithm was suggested based on periodic measurement of Packet Error Rate (PER) and evaluated on a real test-bed in [2]. Yang et al. [8] proposed an analytical model for choice of optimal PCS threshold that includes the impact of a) the bandwidth independent MAC overhead due to PHY header, slot time, etc., and b) bandwidth dependent overhead caused by collisions.

However, a full practical solution for network aggregate throughput optimization must consider the interaction of PCS adaptation with the setting of other parameters, e.g., contention window size, channel rate etc.; this is yet to be achieved. There exists little prior guidance for jointly optimizing aggregate system throughput for a (single channel) multi-rate network with respect to the PCS threshold and link rates in the literature. Earlier link adaptation based approaches such as Auto Rate Fallback (ARF) [10], OAR [11] and Onoe [12] sought to improve the throughput of individual links according to dynamic channel conditions but did not consider use of PCS threshold as a parameter for improving aggregate network performance. Yang et al. [8] showed that for higher PCS threshold and lower data rates, the MAC overhead can decrease and consequently the aggregate one-hop throughput improves; Lin et al. [16] further studied the above tradeoff between spatial reuse and spectrum efficiency with an advanced analytical model. However both these studies were limited to single rate networks with a uniform link distance. In [13], Yang et al. proposed an interesting spatial backoff algorithm which jointly controls the PCS threshold and data rate of individual links. Although the method showed promise in improving spatial reuse, link starvation problems exist when different PCS thresholds are used as shown in [2], [14]. The work closest to our approach in spirit is Zhai et al. [14], who also used a single PCS threshold for variable data rates. However, the data rates in [14] are determined by fixed receiver sensitivities and thus independent of the link distance distribution of a network. In contrast, our range-rate assignment is flexible and allows us the additional degrees of freedom to jointly assign PCS threshold and data rates and achieve the balance between spatial reuse and per-user spectrum efficiency.

In addition to PCS threshold adaptation, transmit power control (TPC), or joint PCS adaptation and TPC can also been used to enhance spatial reuse for 802.11 WLAN, for example [27], [15], [17]. In this work, we assumed all stations use a common transmit power and leave TPC adaptation for future. 


\section{LINK LAYER MODELLING: LITERATURE REVIEW AND COMMENTARY}

The discussion in Section I identifies the four parameters central to CCA in a mesh network: i) node separation distance $D$, ii) transmission range $R_{t r}$, iii) carrier sensing range $R_{c s}$, and iv) interference range $R_{I}$. A survey of the literature on simulation studies of 802.11 ad hoc networks reveals a wide range of choices of the above parameters often without adequate justification which sometimes leads to misconceptions or unexamined assumptions. A further difficulty is the lack of consistency in definitions of the same terms across various authors (notably for $R_{t r}, R_{c s}, R_{I}$ ); hence we first establish definitional clarity and consistency in this regard.

1) Definition 1: The transmission range $R_{t r}$ is defined by a sole Tx-Rx pair in the presence of noise only (i.e., no other concurrent transmission) such that the received power exceeds a signal-to-noise ratio (SNR) threshold $S_{0}$ given by

$$
S_{0}=\frac{P_{r e f} / R_{t r}^{n}}{P_{n}}
$$

where $P_{r e f}$ is the transmit power at a reference distance (from Tx.), which can be given by $\frac{P_{t x} \lambda_{w}^{2}}{16 \pi^{2}}$, where $P_{t x}$ is the transmit power and $\lambda_{w}$ is the wavelength; $P_{n}$ is the additive background noise power and $n$ is the path loss exponent. It is worth emphasizing that $R_{t r}$ denotes the maximum link distance at which packet decoding is possible with high probability at a given link rate.

2) Definition 2: Carrier Sensing Range $R_{c s}$ is defined by

$$
\gamma_{c s}=\frac{P_{r e f}}{R_{c s}^{n}}
$$

i.e., the distance at which the receiver signal power from the source measured by PCS equals the sensing threshold value $\gamma_{c s}$. The implication is that only one among all contending nodes within an area defined by $R_{c s}$ may transmit, and the others defer transmission via the CSMA mechanism in 802.11 DCF whenever the received power as measured by PCS mechanism exceeds $\gamma_{c s}$.

\section{3) Definition 3: Interference Range}

For nominal Tx (S1)-Rx(D1) separation $D$ (where $D<R_{t r}$ ) and ONE interfering concurrent source S2 at a distance DIS from the receiver D1, the signal-to-interference plus noise ratio (SINR) at D1 is given by

$$
\operatorname{SINR}(D)=\frac{P_{r e f} / D^{n}}{P_{n}+P_{r e f} / \mathrm{DIS}^{n}}
$$

The interference range $R_{I}$ is the value of DIS whereby the $\operatorname{SINR}(D)$ equals $S_{0}$, which is given by

$$
R_{I}=\left(\frac{P_{r e f}}{\frac{P_{r e f} / D^{n}}{S_{0}}-P_{n}}\right)^{\frac{1}{n}} .
$$

Equating (1), (4) yields after some simple manipulations

$$
R_{I}=\left(\frac{P_{r e f} / P_{n}}{\left(R_{t r} / D\right)^{n}-1}\right)^{\frac{1}{n}}=\left(S_{0}\right)^{\frac{1}{n}} \frac{R_{t r}}{\left(\left(R_{t r} / D\right)^{n}-1\right)^{\frac{1}{n}}} .
$$

Equation (5) provides a theoretical expression for $R_{I}$ based on the amount of link margin available when $D<R_{t r}$; i.e., only a secondary transmitter within a radius $R_{I}$ of the intended receiver will disrupt the reference transmission (i.e., results in loss of desired packet). It can be shown by taking derivative of (5) that $R_{I}$ is monotonic increasing with $D$, implying that increasing $D \rightarrow R_{t r}$ results in the link becoming more vulnerable to interference as can be expected. Specifically, as $D \rightarrow R_{t r}$, $R_{I} \rightarrow \infty$ implying the loss of all link margin in the limit and hence ANY concurrent transmitter (at whatever distance from the reference receiver) causes the desired packet to be dropped, as the received SINR drops below the threshold needed for packet decoding.

Of particular interest is the fact that for $D \ll R_{t r}, R_{I}$ is approximately proportional to $D$. Note also that in this region, the interference range $R_{I}<R_{t r}$ which belies the folk theorem that $R_{I}$ always exceeds $R_{t r}$.

This is also a good occasion to review and comment on the values of $R_{t r}, R_{c s}, R_{I}$ that have been adopted to date in the literature for network performance evaluation. It is likely that the choice of parameter values used by various authors have been influenced by the default settings in the simulation tools of their choice, e.g., ns-2 and Qualnet. For example, the values used in [5], [7] for experimentation $\left(R_{t r}=250 \mathrm{~m}, R_{c s}=R_{I}=\right.$ $550 \mathrm{~m}$ ) are the default in ns-2 802.11 simulator and may suggest (by incorrect generalization) that $R_{t r} \leq R_{I} \leq R_{c s}$. Alternately, [4] states that " . . for open space environment, the interference range is 1.78 times the transmitter-receiver distance" (and as a corollary, RTS/CTS does not work when $D>\frac{R_{t r}}{1.78}$ ). This fact has been used by other authors subsequently such as [9] for performance studies using the Qualnet simulator.

Both the above assumptions are worthy of closer scrutiny since these have significant impact on network performance. While it is certainly reasonable that $R_{t r}<R_{c s}$ in general, an inequality such as $R_{t r}<R_{I}$ is not true under all conditions, as we show conclusively. Further, assumptions such as $R_{I} \approx 1.78 R_{t r}$ leave (perhaps unwittingly) an impression that $R_{I}$ is typically a constant (2-3) times the transmit range $R_{t r}$, and there exists a linear relationship between the two variables. This is only true in the case $D \ll R_{t r}$; when $D \rightarrow R_{t r}, R_{I}$ increases rapidly to infinity while $R_{t r}$ is fixed! A look at the derivation of the above result reveals the root of the problem; these [4], [27] ignore the presence of background noise and base the interference range on a threshold of purely the received SIR and not received SINR, since it is implicitly assumed that the noise power is negligible compared to the interference power. Such an approximation is only supported when the interference range is small (or equivalently when $D \ll R_{t r}$, i.e., the S-D separation is only a fraction of the transmission range) and not otherwise.

\section{Optimizing Physical CARrier Sensing for HoMOgENEOUS NETWORKS: ANALYTICAL MODEL AND ITS APPLICATION}

\section{A. Analytical Model}

We make the following assumptions for our analytical model in this section:

1) All sources have identical, fixed transmit power. 
2) The channel between any two nodes is identical and nonfading. The mean received signal power is related to the transmit power by a standard propagation power law characteristic $(2 \leq n \leq 6)$.

3) The link distance and capacity is identical for each link.

4) The network is large enough to ignore edge effects and is homogeneous, i.e., it is spatially uniform with a fixed density. Thus the net interference environment as seen at any station is the same, on average.

5) The presence of a hidden terminal impacts aggregate network throughput the same as an exposed node; the latter prevents a successful transmission while the former wastes a transmission opportunity due to a collision.

Let $\mathcal{A}$ denote the set of nodes that are transmitting at time $t$, i.e., the active set within any network. The total received power at any node $i$ (ignoring common constants $P_{r e f}$ ) is given by $P_{R}(i, \mathcal{A})=\sum_{j \in \mathcal{A}} \frac{1}{d(j, i)^{n}}$, where $d(j, i)$ is the distance between nodes $j$ and $i$. The total received power $P_{R}(i, \mathcal{A})$ at a node $i$ represents the effective channel state as observed by $i$. The hidden and exposed node problems are fundamentally caused by the fact that any sender $S$ has only imperfect knowledge of the channel state at its intended receiver $R$. Given a carrier sense threshold $\tau_{c s}$ and an interference power threshold $\tau_{i}$, the sender bases its decision to defer or transmit based on whether $P_{R}(S, \mathcal{A}) \lessgtr \tau_{c s}$ whereas successful packet reception depends on whether $P_{R}(R, \mathcal{A}) \lessgtr \tau_{i}$. There are therefore four cases of interest:

1) $P_{R}(S, \mathcal{A})<\tau_{c s}, P_{R}(R, \mathcal{A})<\tau_{i}$ : In this case, the sender has an opportunity to transmit, and the packet will be received successfully at $\mathrm{R}$ with high probability.

2) $P_{R}(S, \mathcal{A})>\tau_{c s}, P_{R}(R, \mathcal{A})>\tau_{i}$ : In this case, the total received power at $\mathrm{R}$ is too high to allow for successful packet reception, and $\mathrm{S}$ (correctly) does not attempt a transmission.

3) $P_{R}(S, \mathcal{A})<\tau_{c s}, P_{R}(R, \mathcal{A})>\tau_{i}$ : ( $\mathrm{S}$ is "hidden") In this case, the total received power at $\mathrm{R}$ is too high to allow for successful packet reception, but $\mathrm{S}$ may attempt to transmit anyway. The packet will be dropped at $\mathrm{R}$ with high probability.

4) $P_{R}(S, \mathcal{A})>\tau_{c s}, P_{R}(R, \mathcal{A})<\tau_{i}$ : ( $\mathrm{S}$ is "exposed") In this case $\mathrm{S}$ does not attempt a transmission even though a transmission would be received successfully at $\mathrm{R}$ with high probability.

Since the occurrence of hidden and exposed events results in a reduction of throughput, we seek to maximize throughput by minimizing the probability of the hidden and exposed events occurring. Since these events are mutually exclusive we equivalently seek to find the value of $R_{c s}$ (where $\tau_{c s}=1 / R_{c s}{ }^{n}$, $\left.\tau_{c s}=\gamma_{c s} / P_{\text {ref }}\right)$ which minimizes

$$
\begin{aligned}
F\left(R_{c s}\right)=\operatorname{Prob}( & \left.P_{R}(S, \mathcal{A})<\tau_{c s}, P_{R}(R, \mathcal{A})>\tau_{i}\right) \\
+ & \operatorname{Prob}\left(P_{R}(S, \mathcal{A})>\tau_{c s}, P_{R}(R, \mathcal{A})<\tau_{i}\right) .
\end{aligned}
$$

Clearly, the expression for $F$ depends on the active set $\mathcal{A}$ which is unknown in general. We focus on finding the optimal $R_{c s}$ for the case where $\mathcal{A}$ consists of a single node. Alternatively, we could seek to minimize $R_{c s}$ by averaging over all possible $\mathcal{A}$; however we shall show that the optimal $R_{c s}$ found by optimizing for the singleton case matches well the optimal $R_{c s}$ found via

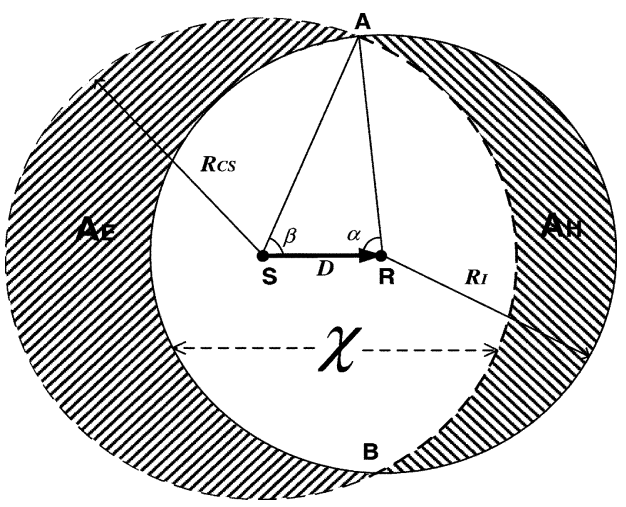

Fig. 1. Hidden and exposed area geometry.

simulations for a random active set. For notational simplicity, we drop $\mathcal{A}$ from expressions such $P_{R}(S, \mathcal{A})$ and simply write $P_{R}(S)$ instead.

When $\mathcal{A}$ consists of a single node, the event that $P_{R}(S, \mathcal{A})>\tau_{c s}$ is equivalent to the node in $\mathcal{A}$ lying inside a circle of radius $R_{c s}$ around S. Similarly the event that $P_{R}(R, \mathcal{A})>\tau_{i}$ is equivalent to the node in $\mathcal{A}$ lying within a circle of radius $R_{I}$ around R. This is illustrated in Fig. 1. In this case, the hidden node event $\left(P_{R}(S)<\tau_{c s}, P_{R}(R)>\tau_{i}\right)$ corresponds to the node in $\mathcal{A}$ lying in the region denoted $A_{H}$ in Fig. 1 which we term the hidden node region. Similarly the exposed node event $\left(P_{R}(S)>\tau_{c s}, P_{R}(R)<\tau_{i}\right)$ corresponds to the node in $\mathcal{A}$ lying in the region denoted $A_{E}$ in Fig. 1 which we term the exposed node region. We also use the notation $A_{H}$ and $A_{E}$ to denote the areas of the hidden and exposed node regions respectively.

Now

$$
\begin{aligned}
& \operatorname{Prob}\left(P_{R}(S)<\tau_{c s}, P_{R}(R)>\tau_{i}\right) \\
& \quad=\operatorname{Prob}\left(P_{R}(S)<\tau_{c s} \mid P_{R}(R)>\tau_{i}\right) \operatorname{Prob}\left(P_{R}(R)>\tau_{i}\right)
\end{aligned}
$$

and similarly

$$
\begin{aligned}
& \operatorname{Prob}\left(P_{R}(S)>\tau_{c s}, P_{R}(R)<\tau_{i}\right) \\
& \quad=\operatorname{Prob}\left(P_{R}(R)<\tau_{i} \mid P_{R}(S)>\tau_{c s}\right) \operatorname{Prob}\left(P_{R}(S)>\tau_{c s}\right) .
\end{aligned}
$$

Since $\mathcal{A}$ consists of a single node, the conditional probability $\operatorname{Prob}\left(P_{R}(S)<\tau_{c s} \mid P_{R}(R)>\tau_{i}\right)$ corresponds to the event that the transmitter is outside carrier sense range of $\mathrm{S}$, given that it is within interference range of $\mathrm{R}$. This is equal to the probability that the transmitter is in the hidden node area given that it is within interference range of $\mathrm{R}$. Assuming that the location of the transmitter is uniformly distributed on a disk of radius $R_{I}$ around R, this is simply $\frac{A_{H}}{\pi R_{I}^{2}}$. Similarly $\operatorname{Prob}\left(P_{R}(R)<\right.$ $\left.\tau_{i} \mid P_{R}(S)>\tau_{c s}\right)$ is $\frac{A_{E}}{\pi R_{c s}{ }^{2}}$. Therefore (6) reduces to

$$
\begin{aligned}
F\left(R_{c s}\right)=\frac{A_{H}}{\pi R_{I}{ }^{2}} \operatorname{Prob}\left(P_{R}(R)>\tau_{i}\right) \\
+\frac{A_{E}}{\pi R_{c s}{ }^{2}} \operatorname{Prob}\left(P_{R}(S)>\tau_{c s}\right) .
\end{aligned}
$$

Now $\operatorname{Prob}\left(P_{R}(R)>\tau_{i}\right)$ is the probability that a node lying within interference range of $\mathrm{R}$ transmits. We invoke the approximation that the probability a node transmits within a region is proportional to the area of the region. This is motivated by the fact that for a homogeneous node distribution, all nodes contend 


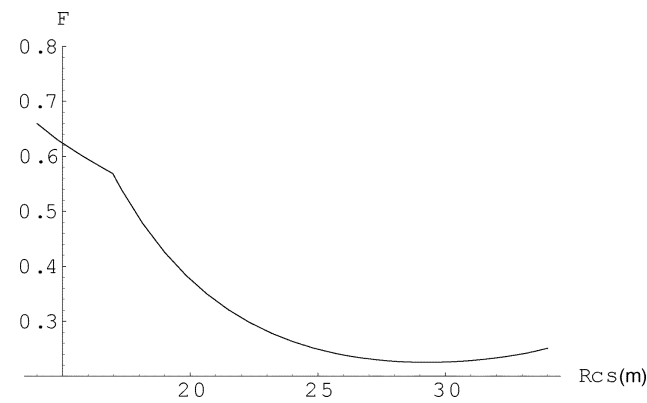

Fig. 2. Probability of hidden or exposed events as a function of $R_{c s}$ for $D=$ $10 \mathrm{~m}$ and $R_{I}=24 \mathrm{~m}$.

for channel have an equal likelihood of winning the channel and the expected number of nodes in a given region is proportional to its area. In addition, the above probability should be in inverse proportion to the area of the carrier sensing region, $\pi R_{c s}{ }^{2}$. This is due to that the simultaneously transmitting nodes will be separated by $R_{c s}$, leading that the density of simultaneously transmitting nodes will be inversely proportional to $R_{c s}{ }^{2}$ in a 2-D network.

With the above, we may express the probability that a node within $R_{I}$ of $R$ transmits as

$$
\begin{aligned}
\operatorname{Prob}\left(P_{R}(R)>\tau_{i}\right) & =\min \left(\frac{c}{\pi R_{c s}^{2}} \pi R_{I}^{2}, 1\right) \\
& =\min \left(1, c \frac{R_{I}^{2}}{R_{c s}^{2}}\right) .
\end{aligned}
$$

Here $c$ is constant $(c \leq 1)$ and its value has no impact on above optimization. Similarly, $\operatorname{Prob}\left(P_{R}(S)>\tau_{c s}\right)=$ $\frac{c}{\pi R_{c s}{ }^{2}} \pi R_{c s}{ }^{2}=c$. Therefore (6) reduces to

$$
F\left(R_{c s}\right)=\frac{A_{H}}{\pi R_{I}^{2}} \min \left(1, c \frac{R_{I}^{2}}{R_{c s}{ }^{2}}\right)+\frac{A_{E}}{\pi R_{c s}{ }^{2}} c .
$$

In order to derive expressions for $A_{H}$ and $A_{E}$, observe that the area $\chi$ (defined by the intersections of the two circles) as shown in Fig. 1 satisfies

$$
A_{E}+\chi=\pi R_{c s}^{2} \quad A_{H}+\chi=\pi R_{I}^{2}
$$

from which it readily follows that $A_{H}-A_{E}=\pi\left(R_{I}^{2}-R_{c s}^{2}\right)$. Thus, it is clear that $A_{H} \lessgtr A_{E} \leftrightarrow R_{I} \lessgtr R_{c s}$.

The area $\chi$ is divided into two regions by the segments AR and RB; let $\chi_{1}$ denote the (area of) the region which includes $S$, and let $\chi_{2}$ denote the (area of) the region that does not include S. We have $\chi=\chi_{1}+\chi_{2}$. Then, from elementary geometry

$$
\chi_{1}=\alpha R_{I}^{2} \quad \chi_{2}=\beta R_{c s}^{2}-2 \Delta S A R
$$

where the angles $\alpha, \beta$ are defined by the triangle $S A R$ and $\triangle S A R$ denotes the area of the triangle. Using the law of cosines

$$
\begin{aligned}
R_{c s}^{2} & =D^{2}+R_{I}^{2}-2 D R_{I} \cos \alpha \\
R_{I}^{2} & =R_{c s}^{2}+D^{2}-2 R_{c s} D \cos \beta
\end{aligned}
$$

and $2 \Delta S A R=D R_{I} \sin \alpha$. Hence

$$
\chi=\alpha R_{I}^{2}+\beta R_{c s}^{2}-D R_{I} \sin \alpha .
$$

Equation (10), (12), and (13) allow us to express $A_{H}$ and $A_{E}$ purely in terms of $R_{c s}, R_{I}$, and $D$. The optimal value of $R_{c s}$

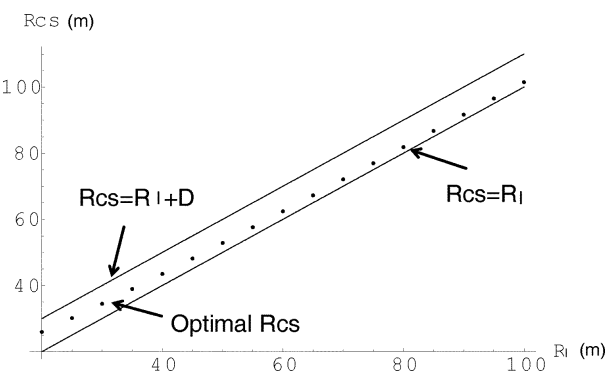

Fig. 3. Optimal $R_{c s}$ as a function of $R_{I}$ for $D=10 \mathrm{~m}$. The two solid lines represent the lines corresponding to $R_{c s}=R_{I}$ and $R_{c s}=R_{I}+D$.

can then be obtained by numerically minimizing the expression in (9).

While determining the exact value of the optimal $R_{c s}$ requires numerical evaluation, we now show that the optimal $R_{c s}$ must lie between $R_{I}-D$ and $R_{I}+D$. Observe from Fig. 1 that if $R_{c s} \geq R_{I}+D$ then $A_{H}=0$. It then follows from (9) that increasing $R_{c s}$ beyond $R_{I}+D$ can only increase $F\left(R_{c s}\right)$. Similarly if $R_{c s} \leq R_{I}-D$, then $A_{E}=0$ and again it follows from (9) that decreasing $R_{c s}$ below $R_{I}-D$ can only increase $F\left(R_{c s}\right)$. Therefore the optimal value of $R_{c s}$ must lie between $R_{I}-D$ and $R_{I}+D$.

It then follows readily that if $D \ll R_{I}$ then the optimal CS range is given by $R_{c s} \approx R_{I}$. Further, as we show later, $R_{c s}=$ $R_{I}$ turns out to be surprisingly robust as well for a variety of network scenarios, i.e., the aggregate throughput at the above point is typically within $5 \%-10 \%$ of the throughput for optimal PCS setting.

\section{B. Numerical Results}

We present numerical results based on computing $F$ in (9). In Fig. 2, $F$ is plotted as a function of $R_{c s}$ for $D=10 \mathrm{~m}$ and $R_{I}=$ $24 \mathrm{~m} .{ }^{3}$ Without loss of generality, constant $c$ is set to 0.5 . The optimal value of $R_{c s}$ is found to be $29.3 \mathrm{~m}$; subsequent OPNET simulation results for a 2-D lattice with 10 m node separation yielded an optimal value of $R_{c s}$ (to within $1 \mathrm{~m}$ granularity) of $29 \mathrm{~m}$.

Keeping $D=10 \mathrm{~m}$ fixed, and varying $R_{I}$ (which corresponds to systems with different SNR requirements, or different path loss exponents), we obtain the dependence illustrated in Fig. 3. This figure confirms that, for $R_{I} \gg D$, the optimal value of $R_{c s} \approx R_{I}$.

Next, we explore the sensitivity of using the above thumb rule, i.e., $R_{c s}=R_{I}$ (which is suboptimal), on the probability of hidden or exposed events. In Fig. 4 we show the normalized deviation $\left(F-F_{\min }\right) / F_{\min }$ of $F$ from its optimal value as a function of $R_{I}$ when $R_{c s}$ is set equal to $R_{I}$. The additional probability of hidden or exposed nodes (over the optimum) induced by this condition is $23 \%$ when $R_{I}=2 D$; this drops to $6 \%$ for $R_{I}=4 D$ as expected, supporting our contention that this may be an adequate setting in many scenarios.

\section{Simulation Evaluation}

In this section, we report results from OPNET v.11 simulations that investigate the effects of modifying the CS range on

\footnotetext{
${ }^{3}$ It is derived from (5) and verified by simulations in [25].
} 


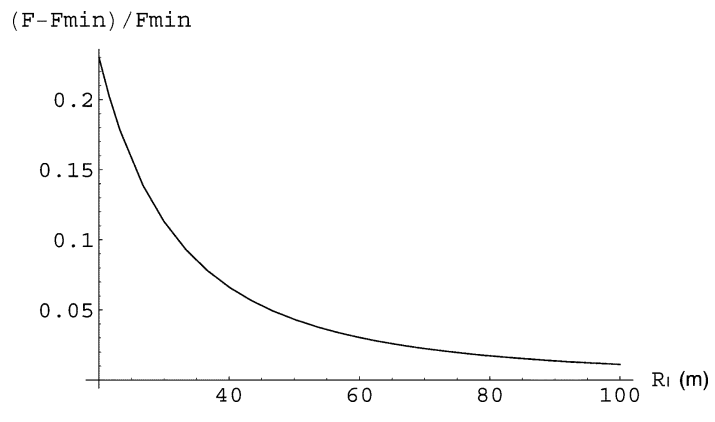

Fig. 4. Deviation of $F$ from its optimal value when $R_{c s}=R_{I}$ and $D=10 \mathrm{~m}$.

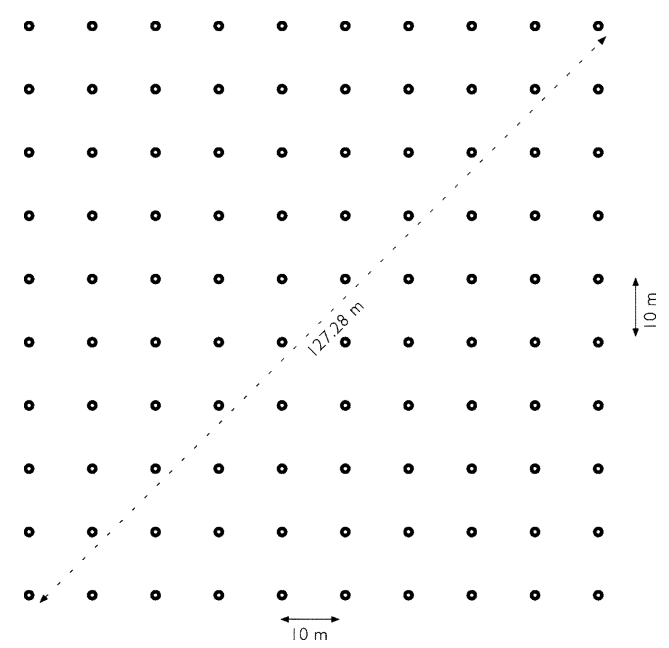

Fig. 5. The $10 \times 10$ grid topology used for OPNET simulations.

the network throughput as a function of various network scenarios. The simulation results will provide values for the optimal PCS threshold setting in more general scenarios than are captured by our analytical model. The simulations were carried using the modified physical carrier sensing module developed in our previous work [25].

1) Simulation Results of a Grid Network: OPNET simulations were run on a $10 \times 10$ square grid of nodes with a grid spacing $D=10 \mathrm{~m}$ as shown in Fig. 5. The reception sensitivity was set such that the reception range was $10 \mathrm{~m}$; thus a node can only receive packets from its "one-hop" neighbors. The physical layer used was $802.11 \mathrm{a}$ at $12 \mathrm{Mbps}$. The carrier sense range $R_{c s}$ was varied from $10 \mathrm{~m}$ to $128 \mathrm{~m} ; R_{c s}=128 \mathrm{~m}$ ensures that all nodes in the simulation are within carrier sense range of each other. A one-hop traffic flow was set up on each edge of the grid in both directions, for a total of 360 flows. Each flow consisted of a Poisson stream of packets generated directly at the IP layer, i.e., no transport protocol was used. The packet size (including IP headers) was a constant 1500 bytes.

For each carrier sense range value, the offered rate of all flows was simultaneously increased until the fraction of offered packets network-wide which got dropped reached $10 \%$. Note that a packet can be dropped for two reasons: either because the MAC layer buffer (which has a capacity of 21 packets) is full and cannot accept another packet from the IP layer, or because the number of retransmission attempts for the packet exceeds the retransmission limit of 7 . We measured the highest offered rate (per flow) for which the packet drop rate stayed below $10 \%$,

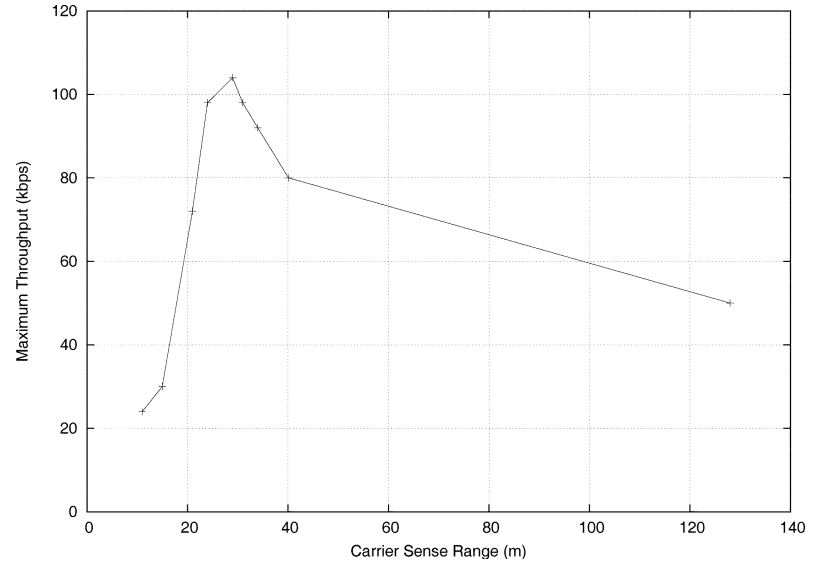

Fig. 6. $T_{\max }$ as a function of $R_{c s}$ from OPNET simulations on a $10 \times 10$ grid.

this is denoted by $T_{\max }$. Since $T_{\max }$ is the per-flow throughput and there are 360 flows in the network totally (considering bi-directional nature of each link), this implies that when the offered traffic per flow is $T_{\max }$, the aggregate traffic network-wide is $0.9 \times 360 \times T_{\max }$.

Note that $T_{\max }$ is an upper bound to the maximum traffic that can be carried simultaneously on each link while maintaining a desired low packet loss rate. We use $T_{\max }$ as our metric for comparison of network performance across various values of $R_{c s}$. Since we increase rates on all links simultaneously, we are essentially considering a "fair" scenario in which all links are used equally. Our interest is in the variation of the throughput with $R_{c s}$, and as such, other choices of metric could also have been be used. In particular, the choice of a packet loss rate of $10 \%$ is somewhat arbitrary; it was chosen to provide a metric that can be measured reliably without excessively long simulation runs. We also chose not to use "saturated" sources, since saturated sources are known to cause unfairness in topologies where not all nodes can sense each other [28].

The maximum value that $T_{\max }$ attains in Fig. 6 is $104 \mathrm{kbps}$ when $R_{c s}=29 \mathrm{~m}$. This optimal carrier sensing range is somewhat higher than the interference range of $24 \mathrm{~m}$ from the results by (5). For $R_{c s}=11 \mathrm{~m}, T_{\max }$ drops significantly to $24 \mathrm{kbps}$. At the other end of the scale, when all nodes can sense each other (i.e., $R_{c s}=128 \mathrm{~m}$ ), $T_{\max }$ drops to $50 \mathrm{kbps}$, which is about $50 \%$ of the maximum achievable rate.

We also noted in the simulations that, for large $R_{c s}$ values, packet losses are almost entirely due to MAC layer buffer overflow. Conversely for low $R_{c s}$ values, packet losses are almost entirely due to the number of retransmissions exceeding the retry limit, and not due to MAC layer buffer overflow.

2) Discussion of Simulation Results: In Fig. 7, we show a source-destination $(\mathrm{S}-\mathrm{D})$ pair in the original $10 \times 10$ grid, along with all the nodes within interference range of $\mathrm{D}$; there are 19 such nodes as shown. The nodes are labeled according to their distance from the sender, i.e., the three nodes labeled "1" are closest to S (at a distance of $10 \mathrm{~m}$ ) and form the first "tier" of potential interferers, while the two nodes labeled "7" are furthest from $\mathrm{S}$ (at a distance of $31.62 \mathrm{~m}$ ) and are the last tier of interferers. As $R_{C S}$ is increased starting from $10 \mathrm{~m}$, additional tiers of potential interferers come within carrier sense range of 


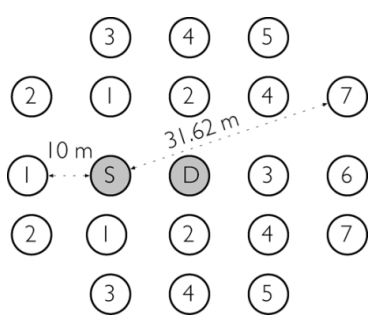

Fig. 7. A source-destination pair and the set of nodes with interference range of $\mathrm{D}$. The nodes are numbered in increasing order of their distance from $\mathrm{S}$. The distances to the closest and farthest tier are shown.

TABLE I

Distance, Number OF HidDEN AND EXPOSED NODES, AND $T_{\max }$ FOR EACH TIER

\begin{tabular}{|c|c|c|c|c|}
\hline Tier & Distance $(\mathrm{m})$ & \# Hidden & \# Exposed & $T_{\max }(\mathrm{kbps})$ \\
\hline 1 & 10.00 & 16 & 0 & 24 \\
\hline 2 & 14.14 & 12 & 0 & 30 \\
\hline 3 & 20.00 & 9 & 1 & 72 \\
\hline 4 & 22.36 & 5 & 5 & 98 \\
\hline 5 & 28.28 & 3 & 7 & 104 \\
\hline 6 & 30.00 & 2 & 10 & 98 \\
\hline 7 & 31.62 & 0 & 16 & 92 \\
\hline
\end{tabular}

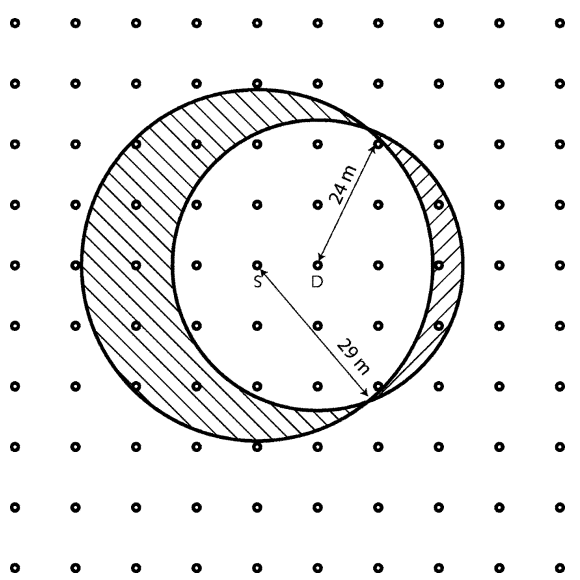

Fig. 8. Hidden and exposed areas for the optimal carrier sensing range in the OPNET simulations. There are three hidden terminals and seven exposed terminals when $R_{c s}$ is set to its optimum value of 29 meters.

$\mathrm{S}$, thus reducing the number of hidden nodes (while increasing the number of exposed nodes). For $R_{c s}>31.62$, all potential interferers are within $\mathrm{CS}$ range of $\mathrm{S}$, thus eliminating hidden nodes entirely.

In Table I, we list the distance of each "tier" of interferers from the sender, the number of hidden and exposed nodes when $R_{c s}$ is set to include that tier but not higher tiers, and the corresponding value of $T_{\max }$. We see that the throughput is maximized when interferers up to the 5th tier are contained within sensing range, leaving three hidden terminals. For the corresponding $R_{c s}$ of $29 \mathrm{~m}$, the sum of the number of hidden and exposed terminals is 10 , which is the minimum possible. Fig. 8 illustrates the hidden and exposed node areas for the optimal $R_{c s}$. At this point, the number of hidden and exposed terminals are not equal. This is due to the optimal CS range is larger than the interference range.

Note that the loss in throughput from setting $R_{c s}=R_{I}$ is about $5.7 \%$, which again supports the conclusion that setting

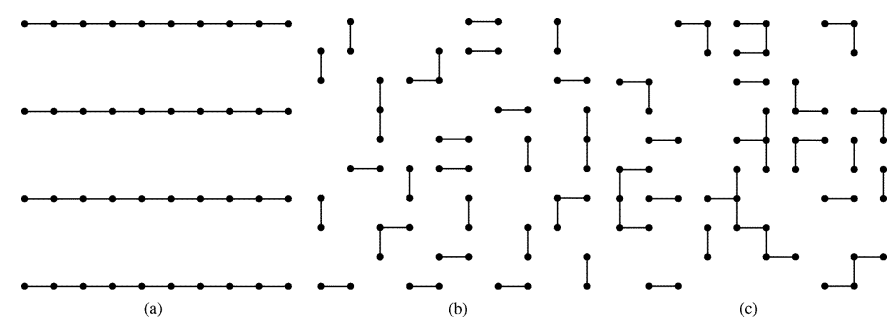

Fig. 9. Three different subsets of the $10 \times 10$ grid.

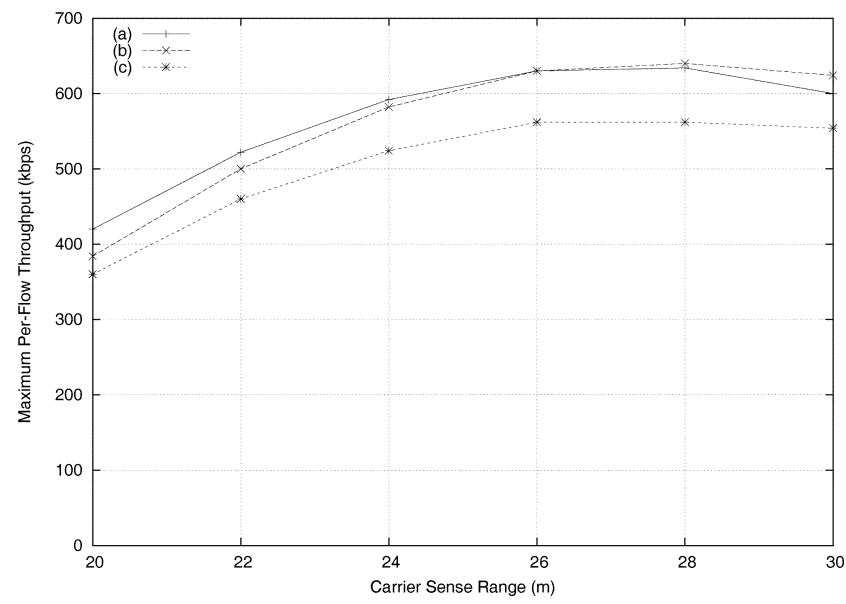

Fig. 10. $T_{\max }$ versus CS range for the three topologies in Fig. 9. In each case, the optimal CS range is $28 \mathrm{~m}( \pm 1 \mathrm{~m})$.

$R_{c s}=R_{I}$ provides a robust close-to-optimal point or a useful initialization for adapting to network conditions at run-time.

3) Robustness of the Result: In this section we consider several other topologies with a view to understanding the limits of applicability of the theoretical results.

Fig. 9 depicts three different subsets of the previous $10 \times 10$ grid. ${ }^{4}$ Each subset contains about 1/6-th the number of links in the original grid. Fig. 10 shows the variation of throughput vs $R_{c s}$ for the three topologies. Although the maximum achievable throughput differs across the three cases, the maximum throughput is attained at nearly the same value of $R_{c s}$ in each case $(=28 \pm 1 \mathrm{~m})$. This shows that the analysis continues to be applicable even when the density of links is significantly reduced.

Next, we consider a 50-pair random mesh network. ${ }^{5}$ The 50 source nodes are located randomly with uniform distribution over a $100 \mathrm{~m} \times 100 \mathrm{~m}$ square area. Each transmitter sends a Poisson stream to its dedicated receiver, which is located in a randomly selected direction with $10 \mathrm{~m}$ separation. All other parameters are the same as the previous experiments. Fig. 11 shows that in this case the optimal value of $R_{c s}$, i.e., $27 \mathrm{~m}$, once again is very close to the analytical estimate $(29 \mathrm{~m})$ validating the robustness of the analytical model for random topologies.

\section{Application of the Analytical Model: RATE-TO-LINK ALLOCATION}

In the analytical model and simulation examples of the previous section, all source-destination distances were identical;

\footnotetext{
${ }^{4}$ These subsets depict the set of co-channel links generated by running various channel assignment algorithms on the $10 \times 10$ grid.
}

${ }^{5}$ This is the same network as [23, Fig. 2(a)]. 


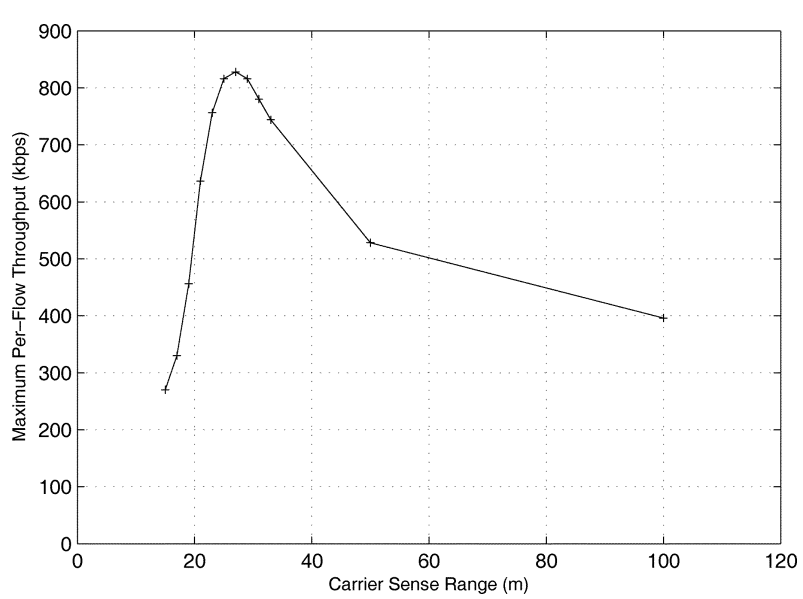

Fig. 11. $T_{\max }$ versus CS range for a random 50-pair mesh network.

in such topologies, a common PCS threshold along with a common data rate for all links is appropriate. However, our work is mainly intended for ad hoc networks where the link (or 1-hop) distance $D$ is a random variable. Therefore, in this section, we investigate the problem of individual link rate assignments according to their distances in a multi-rate network.

In such cases, the average signal to interference ratio at any receive node varies considerably across the network, and accordingly the link capacity. Thus, the optimal PCS threshold or the link data rate should also logically vary across the network as part of any overall strategy for interference management. This would allow us to exploit the available degrees of freedom: different link rates in conjunction with choice of a common PCS threshold to optimize aggregate network throughput. Accordingly, we investigate the problem of individual channel rate assignments to links according to their distances in a multi-rate network. Motivated by the desire to exploit the key result of above analytical model, i.e., $R_{c s} \approx R_{I}$, we propose a link-distance based rate assignment that renders the interference range for all links equal; this in turn allows a single carrier sense range to be used for improving the whole network. ${ }^{6}$

We show the benefit of above rate allocation based on computing $F$ in (9). In Fig. 12, for the schemes with and without rate-range adaptation, $F$ is plotted as a function of link distance $D$. Without rate allocation, data rate $12 \mathrm{Mbps}$ is assumed for all link with different distances, while with rate allocation, the interference ranges of all links are rendered to $24 \mathrm{~m}$. The $R_{c s}$ is set to $29.3 \mathrm{~m}$, the optimal value found in Fig. 2 for $D=10 \mathrm{~m}$ and $R_{I}=24 \mathrm{~m}$. As we can expected, the shorter the links in a system, the greater the decrease in $F$ and consequent greater improvement in aggregate one-hop throughput.

In addition, we will show that the rate assignment rule can be formulated with simple power expressions without the need for knowledge of the path loss exponent. This results in a practical PCS adaptation algorithms, denoted by "PCSadapt" in Section VI.

\footnotetext{
${ }^{6}$ The proposed method is certainly not optimal in general (e.g., a different carrier sense/interference range for each link is feasible), but it affords a simple rate assignment mechanism that nonetheless yields significant improvement in aggregate network throughput compared to the baseline (single rate network) as will be supported by simulation evidence.
}

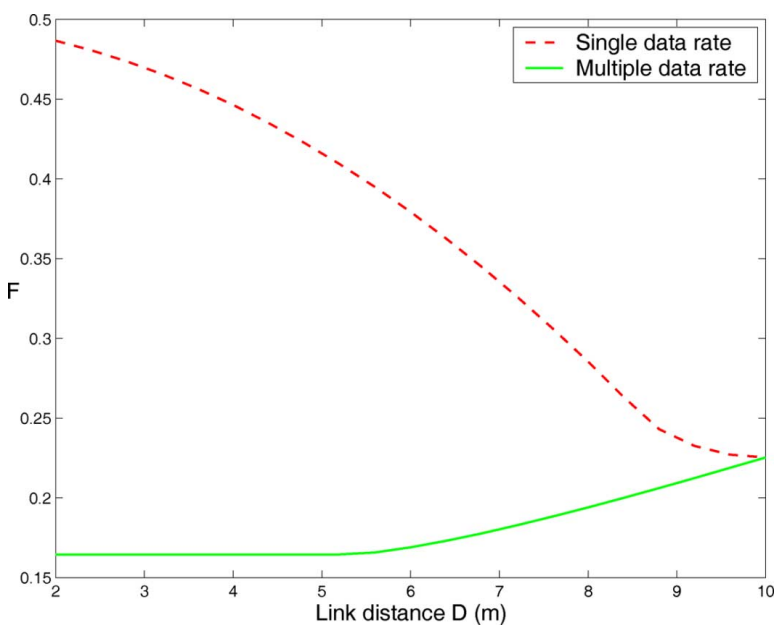

Fig. 12. The benefit of rendering the interference range equal via rate allocation.

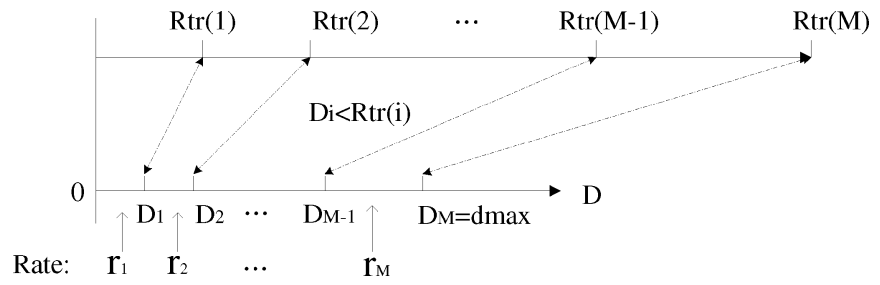

Fig. 13. Principles of rate allocation on individual links.

\section{A. Rate Allocation}

Since the available link rates in IEEE 802.11 are discrete and limited, equalizing the interference range for all links can only be achieved approximately. Let $r_{i}, i=1, \ldots K$ are the available link rates $^{7}$. We assume the ordering $r_{1}>r_{2}>\cdots>r_{K}$ without loss of generality, choose a suitable subset $\left\{r_{1}, \ldots, r_{M}\right\}(M<=K)$ in various examples. We will show that the choice of the the minimum rate $r_{M}$ for the network has significant impact on the aggregate throughput.

As shown in Fig. 13, we assign rates to the individual links as follows: any generic link distance $D$ is divided into $M$ subranges $\left(0=D_{0}, D_{1}\right],\left(D_{2}, D_{3}\right], \ldots\left(D_{M-1}, D_{M}\right]^{8}$. Then if $D \in\left[D_{i-1}, D_{i}\right]$, the corresponding rate $r_{i}$ is selected. In addition, $D_{i}<R_{t r}(i)$, where $R_{t r}(i)$ represents the transmission range corresponding to rate $r_{i}$. Further, if $D<R_{t r}(1)$ and $D_{1}<D<D_{2}$, we assign $r_{2}$ to that link instead of $r_{1}$ implying that the lower rate is preferred.

Thus, using (4), the break-points are

$$
R_{I}=\left(\frac{P_{r e f}}{\frac{P_{r e f} / D_{i}^{n}}{S_{0}(i)}-P_{n}}\right)^{\frac{1}{n}} \quad \text { for } i=1, \ldots M
$$

where $S_{0}(i)$ represents the SNR threshold corresponding to rate $r_{i}$.

Simple manipulations yield

$D_{1}: D_{2}: \cdots: D_{M}=S_{0}(1)^{-1 / n}: S_{0}(2)^{-1 / n}: \cdots: S_{0}(M)^{-1 / n}$.

${ }^{7}$ For 802.11a, $r_{i}$ equals 6, 9, 12, 18, 24, 36, 48 and $54 \mathrm{Mbps}$, i.e., $K=8$.

${ }^{8}$ This implicitly places an upper limit $D_{M}$ on the maximum link distance. 
TABLE II

Benefit of PCS Adaptation Along With Rate Diversity in a Random 50-Pair Mesh With Different Link Distances

\begin{tabular}{|c|c|c|c|}
\hline Scheme & Default $\gamma_{c s}$ with 12Mbps & Optimal $\gamma_{c s}$ with 12Mbps & Optimal $\gamma_{c s}$ with rate allocation \\
\hline Aggregate throughput(Mbps) & 27.8 & 51.7 & 69.4 \\
\hline
\end{tabular}

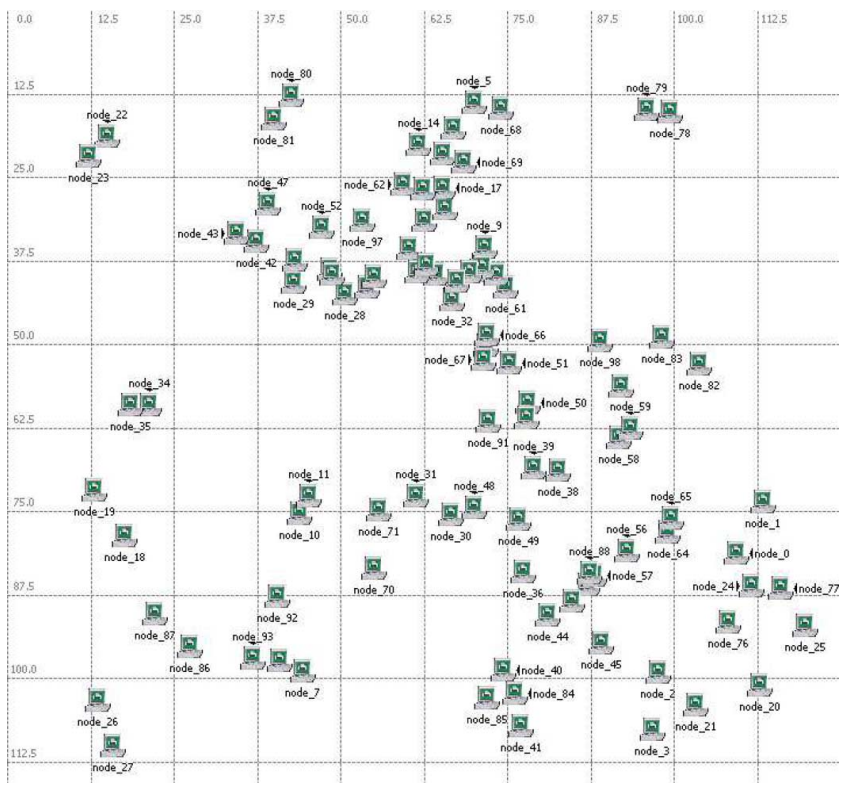

Fig. 14. 50 pairs random high density mesh, wherein node_(2i) sends saturated traffic to its dedicated receiver node_ $(2 i+1)(i=0, \ldots, 49)$.

Consider an illustrative example for $M=6, n=2$ and $r_{1}=54, r_{2}=48, r_{3}=36, r_{4}=24, r_{5}=18$, $r_{6}=12$ Mbps respectively, with maximum link distance $D_{6}=10 \mathrm{~m}$. The link distances are divided into six subranges $\left(0, D_{1}\right],\left(D_{1}, D_{2}\right], \ldots,\left(D_{5}, D_{6}\right]$ corresponding to the six rates $(54,48,36,24,18$ and $12 \mathrm{Mbps})$ respectively. Given $S_{0}(i)=22.06,21.55,16.80,15.04,9.30,7.54 \mathrm{~dB},{ }^{9}$ from (15) we can find

$$
\begin{aligned}
D_{1}: D_{2}: D_{3}: D_{4} & : D_{5}: D_{6} \\
& =0.188: 0.199: 0.3443: 0.422: 0.817: 1,
\end{aligned}
$$

from which $D_{1}=1.88 \mathrm{~m}, D_{2}=0.199 \mathrm{~m}, D_{3}=3.44 \mathrm{~m}$, $D_{4}=4.22 \mathrm{~m}, D_{5}=8.17 \mathrm{~m}, D_{6}=10.0 \mathrm{~m}$.

In practice, instead of directly using link distance for the rate assignment, we assign a data rate according to the measured received signal strength of each link, indicated by RSSI (received signal strength indicator). Rewriting (15), the break-points are related via

$$
\frac{P_{r e f}}{D_{1}^{n}}: \frac{P_{r e f}}{D_{2}^{n}}: \cdots: \frac{P_{r e f}}{D_{M}^{n}}=S_{0}(1): S_{0}(2): \cdots: S_{0}(M)
$$

wherein, the lowest measured link RSSI, $P_{M}$, can be used to set the break-point corresponding to $D_{M}$, i.e., $P_{M}=P_{r e f} / D_{M}^{n}$. Then, denote the power values of other breakpoints with $P_{i}(i=$ $1, \ldots M-1)$, we have

$$
P_{1}: P_{2}: \cdots: P_{M}=S_{0}(1): S_{0}(2): \cdots: S_{0}(M) \text {. }
$$

${ }^{9}$ The SNR thresholds are for 1500 Byte packets at $10 \%$ packet error rate, acquired from OPNET modulation curves. In addition, the $S_{0}$ of 6 and $9 \mathrm{Mbps}$ are 4.53 and $6.29 \mathrm{~dB}$.
In above rate assignment, if the measured RSSI of a link lies between $P_{i-1}$ and $P_{i}$ ( $P_{0}$ is defined as infinity), rate $r_{i}$ will be assigned to the link. Note that the rate assignment does not need to know the $n$ value and thus it can be used directly in the adaptation algorithm proposed in Section VI where $n$ is assumed unknown.

\section{B. Simulation Evaluation}

A random mesh network with random link distances is used to show benefit of PCS adaptation along with rate diversity. In the network shown in Fig. 14, 50 source nodes are located randomly with uniform distribution over a $100 \mathrm{~m} \times 100 \mathrm{~m}$ square area. Each transmitter sends saturated traffic to its dedicated receiver, which is located in a randomly selected direction with uniform $[2,10) \mathrm{m}$ separation distance. We generated several topologies with the same distribution for simulation; only the results from one topology are shown since the results from all others were found to be nearly identical.

The simulation was conducted for 802.11a band with transmit power set to $1 \mathrm{~mW}$ and path loss exponent of 2 . The reception sensitivity was set such that the reception range was 10 $\mathrm{m}$. Both the default and the optimal PCS threshold are studied in the simulation. The minimum rate of the network is set to $12 \mathrm{Mbps}$ and thus from the analytical model, the optimal value of $R_{c s}$ is found to be $29.3 \mathrm{~m}$ for $D=10 \mathrm{~m}$, yielding optimal $\gamma_{c s}=-76.1 \mathrm{dBm}$. The default PCS threshold is set to $-85.8 \mathrm{dBm}$, conservatively set to make the corresponding CS range (i.e., $89 \mathrm{~m}$ ) large enough to eliminate hidden terminals. ${ }^{10}$ In the evaluation, saturated flows are used with a constant packet size 1500 bytes. ${ }^{11}$

Table II shows the simulation results. Compared to default $\gamma_{c s}$, optimal $\gamma_{c s}$ can increase the aggregate throughput by $86 \%$ and, by introducing the proposed rate allocation, can further increase by $34 \%$.

As for individual links, we observed that after PCS adaptation, almost all individual link throughputs increase. However, there may be a few links (typically at network core) whose throughput decrease slightly. This is due to fact that when the network uses a smaller initial $\mathrm{CWmin}$ value (the default $\mathrm{CWmin}$ value of 802.11a is 15) along with a smaller CS range, the links on the edge of the network transmit much more aggressively and cause nodes in the center to sense the channel to be busy more frequently. In order to verify that the slight throughput decrease of the central links is not the source of performance improvement of PCS adaptation, we fixed the contention window size to a large value, 511, and repeated the above simulation. Fig. 15 shows all individual link throughputs. From the figure, we can see that the throughput of all link can be improved by

\footnotetext{
${ }^{10}$ The actual PCS threshold in hardware implementation may vary.

${ }^{11}$ Saturated flows are only used to measure aggregate throughput for 'best effort', while Poisson streams with identical rate are used in Section IV-C to determine the optimal CS range.
} 


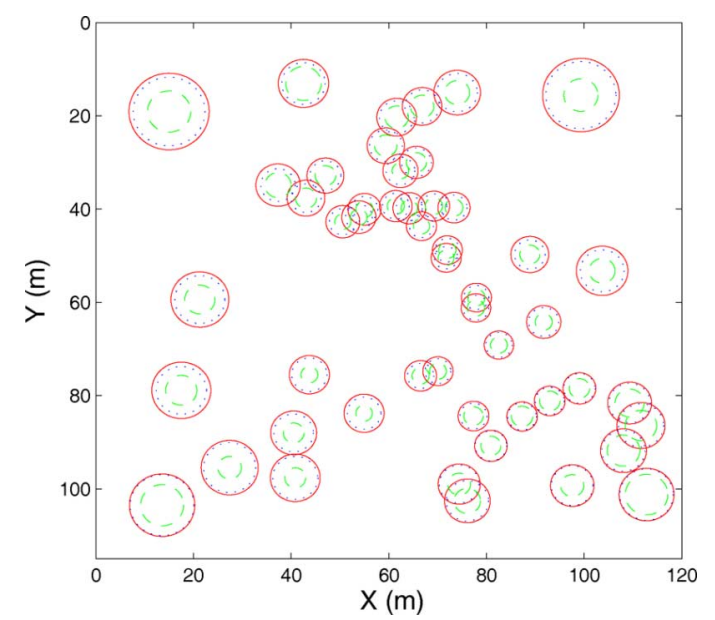

Fig. 15. Link throughput in a 50 pairs random high density mesh. The green dash, blue dot and red solid circles represent the throughputs of individual links with default PCS threshold and $12 \mathrm{Mbps}$, optimal PCS threshold and $12 \mathrm{Mbps}$, and optimal PCS threshold and rate allocation respectively.

PCS adaption and further improved by the proposed rate allocation, ${ }^{12}$ which confirms the effectiveness of both the PCS adaption and rate allocation. Therefore, because in this work we choose to concentrate on optimizing aggregate throughput and the proposed algorithms do not worsen individual link throughputs markedly, we will leave solving the unfairness due to small contention window to the future.

\section{PCS ADAPTATION AlgORITHM FOR High DENSITY HOMOGENEOUS NETWORKS}

In this section, we propose a practical on-line PCS adaptation algorithm for high density (HD) mesh networks where the key parameter in the radio propagation model - the path loss exponent $n-$ is assumed unknown. For known $n$, the analytical model above can be used to predict optimal PCS threshold, denoted by "PCSmodel" in the paper. Given a transmit power and measured link RSSIs, all links distances can be determined; then for any minimum rate, the optimal PCS range (or PCS threshold) can be calculated with the link distances. The "PCSmodel" has already been applied to the example in Section V-B and will be used as a baseline to evaluate the benefits from PCS threshold adaptation in this section.

Variations in $n$ (between 2 and 6) have a significant impact on the optimal PCS threshold. For example, for the minimum rate $12 \mathrm{Mbps}$ and the same receiver sensitivity, the optimal PCS threshold for $n=2$ and $n=4$ differ by $5 \mathrm{~dB}$. Therefore, for the cases when $n$ is unknown, online adaptation algorithm PCS threshold - called "PCSadapt" - has been devised, that uses measured link RSSI for rate assignment and the link PER as an estimate of the effect of hidden terminals to direct PCS threshold adaptation.

In a HD mesh network, the probability of collisions (apart from hidden terminals) is also significant and cannot be neglected. Thus, any adaptation scheme that does not consider collisions can lead to lower-than-optimal aggregate throughput

\footnotetext{
${ }^{12}$ The throughput improvement by rate allocation for links in the right-bottom corner is minor, because these link lengths $\in\left(D_{M-1}, D_{M}\right)$ and their data rates do not increase.
}

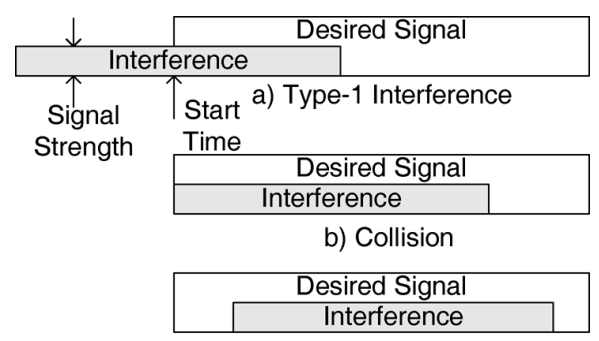

c) Type-2 Interference

Fig. 16. Illustration of different packet losses. The height of each rectangle represents the signal strength of a packet.

or even diverge in extreme conditions. Therefore, a well-designed adaptation algorithm for HD mesh should determine the cause of the packet losses by differentiating measured PER into those resulting from hidden terminals and collisions, respectively. This is difficult because the typical response to a packet transmission is coarse (binary): in ACK based systems, the transmitter only knows success/failure and not the cause of losses. Although there has been several attempts to distinguish the cause of packet losses in wireless networks [19]-[23], as we discussed in [23], there continues to exist a need for low-overhead, robust yet accurate loss differentiation method for IEEE 802.11 HD mesh networks. In our previous work [23], we proposed a novel idea to distinguish and estimate the probability of collision and interference. This method is fully compatible with IEEE 802.11 standard and does not need any physical layer modifications, nor does it incur overhead such as RTS/CTS. The innovation is based on the following insight obtained from extensive OPNET simulations for various data rates, packet sizes, path loss exponents and node densities: the loss probability due to interference was found to be insensitive to CWmin (min. contention window size used for collision resolution) in HD mesh. Based on this observation, a method is proposed to estimate the probability of interference and collision for each individual link and drive PCS threshold adaptation in "PCSadapt" as described next.

\section{A. Estimation of the Effect of Hidden Terminals}

The first step towards an effective real-time PCS adaptation design is to distinguish the causes of packet loss. In this paper, the packet losses are classified into two categories such as collision and interference, which are illustrated in Fig. 16:

1) Collision (Synchronous Interference): one or several concurrent packets start at the same time slot and they cause the desired packet to be corrupted. Collision events are denoted by $\mathbf{C}$.

2) (Asynchronous) Interference 1: In the absence of $\mathbf{C}$, if the received signal strength prior to the desired packet arrival is strong enough to cause the desired packet to be dropped; this event is considered as Type- 1 Interference, and is denoted by I1.

3) (Asynchronous) Interference 2: In the absence of $\mathbf{C}$ and I1, the sum of one or more subsequent arriving packets can cause the desired packet to be corrupted; this is Type- 2 Interference and is denoted by $\mathbf{I} 2$.

Note that asynchronous link-layer interference has been subdivided into two sub-categories: event I1 refers to inference prior while event 12 denotes interference after arrival of desired 
packet. Both of them are referred as hidden terminal problem in the literature. The event due to asynchronous interference, including both $\mathrm{I} 1$ and $\mathrm{I} 2$, will be denoted by $\mathbf{I}$.

Based on the discovery with extensive simulations "changing CWmin has minor effect on the PER due to I for each link", a method for loss differentiation is devised in the previous work [23], which can estimate the PER due to I, $\operatorname{Pr}\{I\}$, and the PER due to $\mathrm{C}, \operatorname{Pr}\{C\}$, for each individual link with default CWmin (Cwmin $=15$ in 802.11a). Such information will be used in the adaptation algorithm.

During the estimation, the whole network will be forced to use a large $\mathrm{CWmin}$ (e.g., 127) to minimize $\operatorname{Pr}\{C\}$. Then, the observed PER for large CWmin can be assumed to equal $\operatorname{Pr}\{I\}$. Finally, $\operatorname{Pr}\{C\}$ for each link can be acquired by subtracting $\operatorname{Pr}\{I\}$ from the total PER with the default CWmin.

\section{B. PCS Adaptation Algorithm Based on Loss Differentiation}

The "PCSadapt" algorithm adaptively searches for the optimal PCS threshold (maximize aggregate throughput) while satisfying the maximum PER (interference) constraint on each link. "PCSadapt" is an extension of the previous centralized PCS adaptation algorithm in [2] and uses additional statistical information of differentiated packet loss rate; while [2] uses the total link PER.

In "PCSadapt", algorithm run-time is divided into two segments: adaptation and normal operation. The adaptation segment is the period during when the proposed algorithm updates the PCS threshold while forcing all network nodes to use a large CWmin. Such adaptation can be performed on a fixed schedule such as the first several minutes in each hour. After determining the suitable PCS threshold at the end of the adaptation, all stations use the default $\mathrm{CWmin}$ value during the normal (data transfer) operation.

A schematic block diagram of "PCSadapt" is shown in Fig. 17 and we define the following:

- $i$ : iteration index corresponding to PCS threshold updating period

- T: PCS threshold updating period

- $T_{a}$ : adaptation segment, $T_{a}=k T, k \in N$

- $P_{m}(i)$ : the PER of the link with highest PER within $i$ th PCS threshold updating period with the large CWmin

- $p_{\min }, p_{\text {max }}$ : target minimum, maximum PER

- $\gamma_{c s}(i)$ : PCS threshold used after $i$ th PCS threshold update

- $\delta$ : PCS adaptation step

- $\gamma_{\min }, \gamma_{\max }:$ minimum, maximum PCS threshold

- $r_{M}$ : the minimum rate

- $P_{M}$ : the lowest link RSSI

- $P_{1}, P_{2}, \ldots, P_{M}$ : subrange boundaries in power

- $r_{1}, r_{2}, \ldots, r_{M}$ : the rates of each subrange

Structurally, "PCSadapt" operates as follows: at the beginning of the adaptation segment, a central server collects all link RSSI for processing and broadcasts the new subrange boundaries to all stations. Here, given an input minimum rate, the subrange boundaries are determined by (17). Then all links set their data rates accordingly, and the rates are kept fixed throughout the adaptation and normal operation segments (on the assumption that our application is intended for largely static nodes).

During the adaptation segment, all stations measure the perlink PER (the ratio between the number of received ACK and

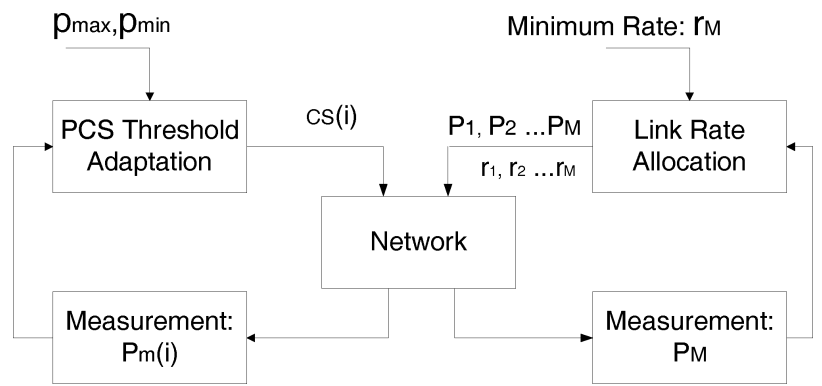

Fig. 17. The block diagram of "PCSadapt" for on-line PCS adaptation with multiple data rates.

the number of transmitted data packets within a specific time duration, $T)$. The PER of the link with highest value, $P_{m}(i)$, will be used for adapting the PCS threshold for the next operation segment based on the following:

$$
\gamma_{c s}(i)= \begin{cases}\max \left(\gamma_{c s}(i-1)-\delta, \gamma_{\min }\right), & \text { if } P_{m}(i)>p_{\max } \\ \min \left(\gamma_{c s}(i-1)+\delta, \gamma_{\max }\right), & P_{m}(i)<p_{\min } \\ \gamma_{c s}(i-1), & \text { otherwise. }\end{cases}
$$

Note that the information exchanges are only performed around each updating period within adaptation segment, so the communication overhead is expected to be minor.

\section{Simulation Evaluation}

1) Simulations Set-up: Altogether two types of network topologies are investigated respectively: a) random mesh network; b) random multi-cell HD WLAN. The former can be assumed as a one-tier mesh or the tier-2 (the backhaul AP-AP mesh) of a two-tier mesh ${ }^{13}$ [24]; while the latter can be assumed as the tier-1 (client-AP connectivity) of a two-tier mesh, where each AP serves multiple tier-1 clients which form a basic service set (BSS). In each scenario, all links work on a single 802.11a channel and all traffics are assumed to be saturated flows with a constant packet size 1500 bytes.

For averaging the PER, $T$ is set to $10 \mathrm{~s}$. The reception sensitivity was set such that the reception range was $10 \mathrm{~m}$; thus a receive node can only receive packets up to a maximum distance of $10 \mathrm{~m}$. We set the target PER ranges such as $\left(p_{\min }, p_{\max }\right)=$ $(0.05,0.1),(0.1,0.2),(0.2,0.3)$ or $(0.3,0.4), \delta=1 \mathrm{~dB}$ and $\left(\gamma_{\min }, \gamma_{\max }\right)=(-85.8 \mathrm{dBm},-66.8 \mathrm{dBm})$ for the PCS adaptation. Here, $\gamma_{\max }$ was set close to the reception sensitivity; while $\gamma_{\min }$ was to a typical value of PCS threshold in current hardware, which makes the CS range large enough to minimize $\operatorname{Pr}\{I\}$. The initial PCS threshold for each $\left(p_{\min }, p_{\max }\right)$ is set to $\gamma_{\max }$. For each $\left(p_{\min }, p_{\max }\right)$, the PCS adaptation algorithm will run for $T_{a}=200 \mathrm{~s}(k=20)$ with the initial PCS threshold. Thus there will be 20 PCS updating periods for each $\left(p_{\min }, p_{\max }\right)$. The simulation will continue with the finalized PCS thresholds as a result of the adaptation and the default CWmin value for another $100 \mathrm{~s}$ (duration chosen based on traffic patterns) to accurately measure network performance post PCS adaptation.

2) Simulation Results and Discussion: First, a 50-pair random mesh network but with identical link distances is used to demonstrate "PCSadapt" and the resulting improvement in spatial reuse compared to earlier adaptation methods such

\footnotetext{
${ }^{13}$ The two tiers are usually separated in the frequency domain, i.e., using $802.11 \mathrm{a}$ or b/g respectively.
} 


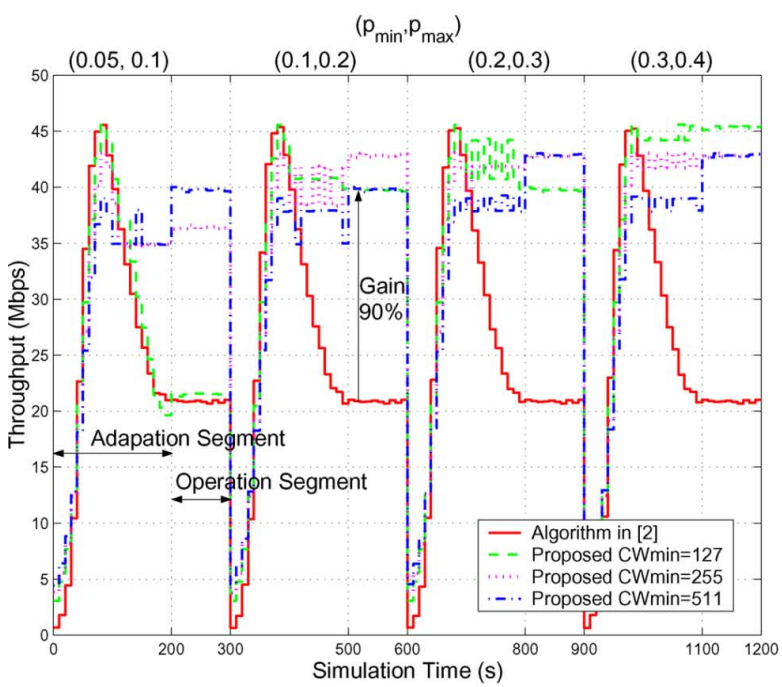

(a) Average throughput

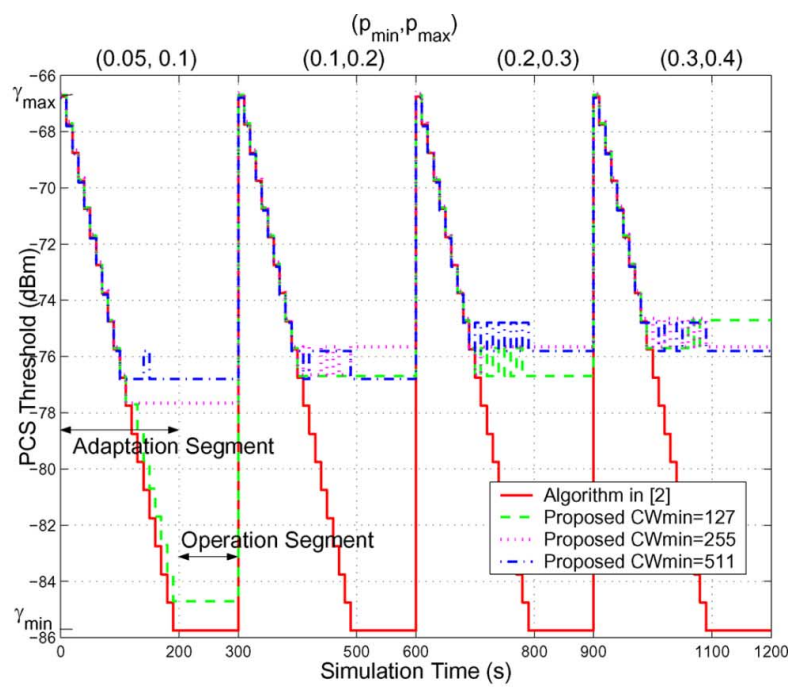

(b) Tracing the PCS threshold

Fig. 18. PCS adaptation in random 50-pair mesh network.

as [2]. The nodes obey the same uniform distribution as the one in Fig. 14 except that all link distances are fixed at 10 m. Each transmitter sends saturated traffic to its dedicated receiver, which is located in a randomly selected direction. In the simulation, we used a fixed data rate $12 \mathrm{Mbps}$, transmit power of $1 \mathrm{~mW}$ and path loss exponent equal to 2 .

Fig. 18 shows average throughput and changes of PCS threshold in the random 50-pair mesh network with our proposed "PCSadapt" algorithm. The results by [2] are compared with "PCSadapt". For "PCSadapt", three different CWmin values such as 127, 255 and 511 were investigated respectively in the estimation for $\operatorname{Pr}\{I\}$ and carrier sensing adaptation.

From Fig. 18(b), PCS threshold (solid line) with the algorithm in [2] cannot converge even for a high target PER range like $(0.3,0.4)$ and will keep on decreasing to the minimum value $\gamma_{\min }=-85.8 \mathrm{dBm}$ (the value in legacy network without PCS adaptation). This is due to fact that this algorithm ignores the PER due to collision, $\operatorname{Pr}\{C\}$. As we discussed in [23], decreasing PCS threshold does not lower the PER due to collision and may even increase it. Thus any PCS threshold will not satisfy the maximum PER constraint and will be forced to decrease via adaptation. Finally the network will use a very unnecessarily low PCS threshold and the achievable spatial reuse will be greatly reduced.

By contrast, our proposed algorithm for the target PER range allows the PCS threshold to converge to a close-to-optimal value for throughput maximization. The optimal PCS threshold is predicted to be $-76.1 \mathrm{dBm}\left(R_{c s}=29.3 \mathrm{~m}\right)$ by the model in Section IV. Therefore, comparing with [2], the aggregate operational throughput was increased by more than $90 \%$ (from 21 Mbps to $40 \mathrm{Mbps})$. The one exception where our algorithm did not converge is the case with a target PER range $(0.05,0.1)$ and CWmin $=127$. In this case, $\operatorname{Pr}\{C\}$ of the worst link with CWmin $=127$ exceeds $10 \%$, which suggests that in order to assure convergence, the target PER range should be adjusted upwards. In addition, as shown in Fig. 18, a larger target PER range tends to lead to a higher (more aggressive) PCS threshold and higher aggregate throughput. However, the higher throughput also results in increased PER of the worst link and therefore,
TABLE III

PCS ADAPTATION IN A RANDOM 50-PAIR MESH With DIFFERENT LINK DISTANCES

\begin{tabular}{|c|c|c|}
\hline & Throughput(Mbps) & PCS threshold $(\mathrm{dBm})$ \\
\hline Legacy(12) & 27.8 & -85.8 \\
\hline PCSmodel(12) & 69.4 & -76.1 \\
\hline PCSadapt(12) & 71.8 & -75.8 \\
\hline
\end{tabular}

the selection of the target PER range should consider the above tradeoffs accordingly.

Second, we use the random mesh network in Fig. 14 to study how "PCSadapt" performs when link distance are different. In this experiment, PER ranges was set to $\left(p_{\min }, p_{\max }\right)=(0.1,0.2)$ and the large $\mathrm{CWmin}$ value is 511. As a baseline, we use "Legacy $(\mathrm{x})$ " to indicate a fully non-adaptive network whose PCS threshold is fixed at the default PCS threshold for the fixed data rate $\times$ Mbps; whereas "PCSmodel(x)" and "PCSadapt(x)" indicates the solution of "PCSmodel" and "PCSadapt" for a adaptive PCS threshold network where the link rates are variable, but with minimum rate equal to $\mathrm{x}$ Mbps as above.

Table III shows the simulation results for the minimum rate 12 Mbps. The table indicates that comparing "Legacy(12)", "PCSadapt(12)" increases the aggregate throughput by $159 \%$. The above improvements indicate the effectiveness of the PCS adaptation along with the proposed rate allocation. In addition, the final PCS threshold after adaptation is very close $(0.3 \mathrm{~dB}$ difference) to that in "PCSmodel(12)", predicted by the analytical model in Section IV. It implies that with our rate allocation principle, "PCSadapt(12)" based on differentiated PER also converges to an optimal PCS value for throughput maximization.

Third, another dense WLAN scenario (co-channel cells in a two-tier mesh) with a higher path loss exponent is studied, shown in Fig. 19. It comprises 25 co-channel cells with cell radius of 10 meters and AP-to-AP distance of 20 meters. Each cell has one AP and one client (STA), and both APs and clients are transmitting saturated UDP traffic, i.e., a total of 50 links in the network. The link distances are uniform $[2,10) \mathrm{m}$. In this experiment, path loss exponent is changed to a higher value 3 and 


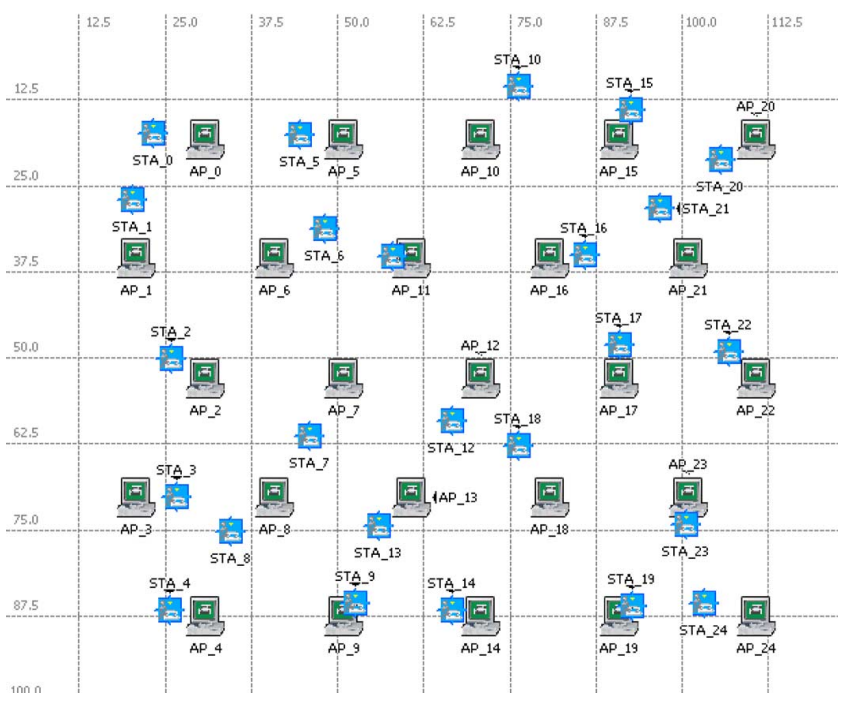

Fig. 19. A 25-cell random WLAN.

thus the transmit power is increased to $25 \mathrm{~mW}$ to compensate for the higher attenuation. All other parameters are the same as the previous experiment.

Table IV shows the simulation results for the three minimum rates: 6, 12 and 24 Mbps. The optimal PCS thresholds used in "PCSmodel" for the three rates are $-72.6,-74.1$, and $-79.3 \mathrm{dBm}$, respectively. As shown in the table, the differences between the above values and finalized PCS threshold $(-71.8$, -72.8 , and $-79.8 \mathrm{dBm}$ ) are all less than $1.5 \mathrm{~dB}$. At convergence, the PCS threshold values translates in significant gains of $381 \%, 243 \%, 84 \%$ respectively, in aggregate throughput via "PCSadapt(x)" compared to "legacy (x)". Moreover, for the minimum rate 6 and 12, it seems that the "optimal" PCS thresholds predicted by the analytical model are slightly conservative (by about 10\%) in throughput compared with "PCSadapt(x)". This highlights the necessity for on-line algorithm in general, as the sensitivity of the analytical model to network topology leads to variable predictions.

Note further that the aggregate throughput "PCSadapt(12)" is much higher than those of "PCSadapt(6)" and "PCSadapt(24)". This underscores the fact that the choice of the minimum rate in a network is fundamental to the tradeoff between spatial reuse and spectrum efficiency. Spatial reuse indicates the total number of simultaneous co-channel links that can operate in a given network, and spectrum efficiency determines the throughput of each link; the aggregate one-hop network throughput is the product of the two. Ideally, we should maximize both but they are at odds, as can be explained by the following: given a link distance, choosing a higher link data rate (increasing spectrum efficiency) increases the interference range. Thus, a larger carrier sensing range (achieved with lower PCS threshold) is needed to protect the link from interference, which in turn leads to less spatial reuse. Therefore, achieving optimal interference management calls for tuning the CS range in conjunction with the available link rates. A more thorough investigation of jointly adaptive selection of link rates and PCS threshold will be left for future work.

Finally, we investigate the impact of channel fading with the proposed PCS adaptation on the network performance. The
TABLE IV

PCS ADAPTATION IN A 25-CELL WLAN WiTH DIFFERENT LINK DISTANCES

\begin{tabular}{|c|c|c|}
\hline & Throughput(Mbps) & PCS threshold (dBm) \\
\hline Legacy(6) & 21.3 & -85.8 \\
\hline PCSmodel(6) & 94.6 & -72.6 \\
\hline PCSadapt(6) & 102.5 & -71.8 \\
\hline Legacy(12) & 37.3 & -85.8 \\
\hline PCSmodel(12) & 114.2 & -74.1 \\
\hline PCSadapt(12) & 127.8 & -72.8 \\
\hline Legacy(24) & 55.3 & -85.8 \\
\hline PCSmodel(24) & 106.2 & -79.3 \\
\hline PCSadapt(24) & 102.0 & -79.8 \\
\hline
\end{tabular}

TABLE V

PCS ADAPTATION IN A RANDOM 50-PAIR MESH With LOGNORMAL FADING MODEL

\begin{tabular}{|c|c|c|}
\hline & Throughput(Mbps) & PCS threshold (dBm) \\
\hline Legacy(12) & 20.5 & -85.8 \\
\hline PCSadapt(12) & 36.7 & -77.8 \\
\hline
\end{tabular}

50-pair random mesh network used in the first example of this section will be evaluated again with Lognormal fading added to model short-term channel fluctuations. The received signal strength of each packet $P_{r x}$, on a link is now given by

$$
P_{r x}=P_{r e f} / D^{n}+X(\text { all in } \mathrm{dB})
$$

where $X$ is a Gaussian random variable with zero mean and variance $\sigma^{2}$. However, evaluating performance in small-scale or fast fading (i.e., $X$ is randomly chosen every few milliseconds) will dramatically increases the complexity of the simulation. Instead, we assume that the lognormal fading level is constant over a packet duration. We set the $\sigma=1.5$ which matches the experimental value found in [2]. In addition, PER range for PCS adaptation is set to $\left(p_{\min }, p_{\max }\right)=(0.1,0.2)$ and the large CWmin value used in the adaptation segment is 511 . Table $\mathrm{V}$ shows the simulation results. We observe that with channel fading, the algorithm "PCSadapt(12)" still converges and achieves a much higher throughput than "Legacy" ( $>179 \%)$, suggesting the effectiveness and robustness of the proposed algorithm even for slowly fading scenarios.

\section{CONCLUSION}

We have presented an analytical model for choosing the PCS threshold in 802.11 mesh network to optimize the aggregate throughput. The validity of this result was investigated by OPNET simulations. The robustness of the analysis to a range of network scenarios was undertaken and shows the validity of using $R_{c s} \approx R_{I}$ as a robust close-to-optimal setting or a useful initialization.

This key result then motivated a rate-to-link allocation method for more pragmatic network topologies with non-uniform link distances, that is, rendering the interference range for all links equal. This in turn allows a single carrier sense range to be used for improving the whole network.

Finally, we proposed a practical PCS adaptation algorithm to achieve the benefit of tunable PCS threshold in real time. The proposed algorithm is based on LD, which can estimate PER due to hidden terminals and collisions respectively. The former are used as the measurement of hidden terminal effect 
for PCS adaptation. Extensive simulation results show that the PCS adaptation along with the rate-to-link allocation can always make the PCS threshold converge to its optimal value and thus outperform schemes without PCS adaptation and adaptation schemes without LD by more than $90 \%$ in terms of the aggregate throughput.

In the future work, we will improve the fairness in the PCS adaptation and investigate the tradeoff between spatial reuse and spectrum efficiency in the joint PCS threshold and data rate allocation.

\section{REFERENCES}

[1] J. Zhu, X. Guo, L. L. Yang, W. S. Conner, S. Roy, and M. M. Hazra, "Adapting physical carrier sensing to maximize spatial reuse in 802.11 mesh networks," Wiley J. Wireless Commun. Mobile Comput., vol. 4, pp. 933-946, 2004.

[2] J. Zhu, B. Metzler, X. Guo, and Y. Liu, "Adaptive CSMA for scalable network capacity in high-density WLAN: A hardware prototyping approach," in Proc. IEEE INFOCOM, 2006.

[3] F. Ye, S. Yi, and B. Sikdar, "Improving spatial reuse of IEEE802.11 based ad hoc networks," in Proc. IEEE Globecom, San Francisco, CA, Nov. 2003.

[4] K. Xu, M. Gerla, and S. Bae, "How effective is the IEEE 802.11 RTS/CTS handshake in ad hoc networks," in Proc. IEEE Globecom, Taipei, Taiwan, Nov. 2002.

[5] G. Anastasi, E. Borgia, M. Conti, and E. Gregori, "WiFi in ad hoc mode: A measurement study," in Proc. 2nd IEEE Conf. Pervasive Computing \& Communication, 2004.

[6] Intel Pro/Wireless 2200BG Driver for Linux, [Online]. Available: http://ipw2200.sourceforge.net

[7] P. C. Ng and S. C. Liew, "Offered load control in IEEE802.11 multi-hop ad hoc networks," in Proc. 1st IEEE Conf. Mobile Ad-Hoc and Sensor Systems, Ft. Lauderdale, FL, 2004.

[8] X. Yang and N. H. Vaidya, "On the physical carrier sense in wireless ad hoc networks," in Proc. IEEE INFOCOM, Miami, FL, Mar. 2005.

[9] A. Vasan, R. Ramjee, and T. Woo, "ECHOS: Enhanced capacity 802.11 hotspots," in Proc. IEEE INFOCOM, Miami, FL, Mar. 2005.

[10] A. Kamerman and L. Montaban, "Wavelan-II: A high-performance wireless LAN for the unlicensed band," Bell Systems Tech. J., 1997.

[11] B. Sadeghi, V. Kanodia, A. Sabharwal, and E. Knightly, "OAR: An opportunistic auto-rate media access protocol for ad hoc networks," ACM/Kluwer Mobile Networks and Applications Journal (MONET): Special Issue on Selected Papers from MobiCom 2002.

[12] Madwifi Project, , 2005 [Online]. Available: http://madwifi.sourceforge.net

[13] X. Yang and N. Vaidya, "Spatial Backoff Contention Resolution for Wireless Networks", Technical Report Univ. of Illinois, Urbana-Champaign, 2006.

[14] H. Zhai and Y. Fang, "Physical carrier sensing and spatial reuse in multirate and multihop wireless ad hoc networks," in Proc. IEEE INFOCOM, 2006.

[15] T. S. Kim, H. Lim, and J. Hou, "Improving spatial reuse through tuning transmit power, carrier sense threshold, and datarate in multihop wireless networks," in Proc. ACM Mobicom, Sep. 2006.

[16] T. Y. Lin and J. C. Hou, "Interplay of spatial reuse and SINR-determined data rates in CSMA/CA-based, multi-hop, multi-rate wireless networks," in Proc. IEEE INFOCOM, May 2007.

[17] J. Fuemmeler, N. H. Vaidya, and V. V. Veeravalli, "Selecting Transmit Powers and Carrier Sense Thresholds for CSMA Protocols", Technical Report, University of Illinois at Urbana-Champaign,, Oct. 2004.

[18] J. Kim, S. Kim, S. Choi, and D. Qiao, "CARA: Collision-aware rate adaptation for IEEE 802.11 WLANs," in Proc. IEEE Conf. Computer Communications, Apr. 2006.

[19] J. H. Yun and S. W. Seo, "Collision detection based on transmission time information in IEEE 802.11 wireless LAN," in IEEE Pervasive Computing and Communications Workshops, 2006, Mar. 2006.

[20] K. Whitehouse, A. Woo, F. Jian, J. Polastre, and D. Culler, "Exploiting the capture effect for collision detection and recovery," in Proc. IEEE Em-NetS-II Workshop, May 2005.

[21] Q. Pang, S. C. Liew, and V. C. M. Leung, "Design of an effective lossdistinguishable MAC protocol for 802.11 WLAN," IEEE Commun. Lett., Sep. 2005.

[22] D. Malone, P. Clifford, and D. J. Leith, "MAC layer channel quality measurement in 802.11," IEEE Commun. Lett., Feb. 2007.

[23] H. Ma, S. Shin, and S. Roy, "Optimizing throughput with carrier sensing adaptation for IEEE 802.11 mesh networks based on loss differentiation," in Proc. IEEE ICC, 2007.

[24] S. Roy, A. Das, R. Vijayakumar, H. Alazemi, H. Ma, and E. Alotaibi, "Capacity scaling with multiple radios and multiple channels in wireless mesh networks," Proc. IEEE WiMesh, Sep. 2005.

[25] H. Ma, E. Alotaibi, and S. Roy, "Analysis and simulation model of physical carrier sensing in IEEE 802.11 mesh networks," in Proc. OPNETWORK Conf. 2006, Washington, DC, Aug. 2006.

[26] IEEE Standard for Wireless LAN Medium Access Control: MAC and PHY Specifications, ISO/IEC 8802-11, 1999(E), Aug. 1999.

[27] Y. Zhou and S. M. Nettles, "Balaning the hidden and exposed node problems with power control in CSMA/CA-based wireless networks," in Proc. WCNC, 2005.

[28] X. Wang and K. Kar, "Throughput modelling and fairness issues in CSMA/CA based ad hoc networks," in Proc. IEEE INFOCOM, 2005.

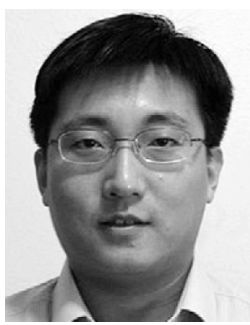

Hui Ma (S'03) received the B.S. and M.S. degrees, both in electronic engineering, from Tsinghua University, Beijing, China, in 2001 and 2004, respectively.

Since September 2004, he has been working towards the Ph.D. degree in electrical engineering at the University of Washington, Seattle. His main research interests are wireless LAN, wireless ad hoc networks and mobile wireless networks. His current work is on MAC design for multi-hop ad hoc networks.

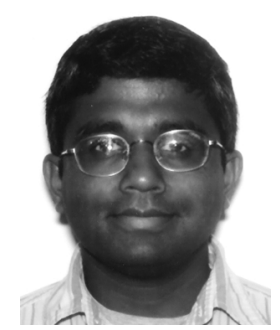

Rajiv Vijayakumar received the B.Tech. degree from the Indian Institute of Technology, Bombay, in 1994, the M.S. degree from Louisiana State University in 1997, and the Ph.D. degree from the University of Michigan in 2004, all in electrical engineering. CA

$\mathrm{He}$ is currently with Qualcomm Inc., San Diego,

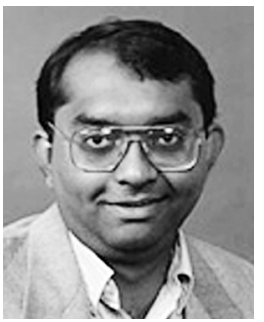

Sumit Roy (F'07) received the B.Tech. degree from the Indian Institute of Technology, Kanpur, in 1983, and the M.S. and Ph.D. degrees from the University of California at Santa Barbara, all in electrical engineering, in 1985 and 1988, respectively, as well as an M.A. in statistics and applied probability in 1988 .

His previous academic appointments were at the Moore School of Electrical Engineering, University of Pennsylvania, and at the University of Texas, San Antonio. He is presently Professor of Electrical Engineering at the University of Washington where his research interests include analysis and design of communication systems and networks, with a topical emphasis on next-generation mobile and wireless networks.

Dr. Roy's activities for the IEEE Communications Society include membership of several technical committees, and he presently serves as an Editor for the IEEE TRANSACTIONS ON COMMUNICATIONS.

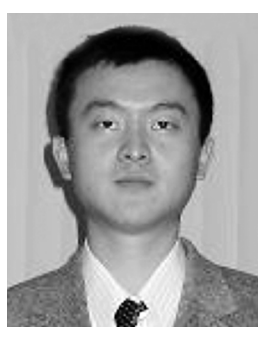

optimization.
Jing Zhu (M'05) received the B.S. and M.Sc. degrees, both in electronic engineering, from Tsinghua University, Beijing, China, in 2000 and 2001, respectively, and the Ph.D. degree in electrical engineering from the University of Washington, Seattle, in 2004.

Currently, he is a research scientist in the Communications Technology Lab, Intel Corporation, Hillsboro, OR. His main research interest is high-performance wireless networking and its applications, e.g., Internet-on-Move, Digital Home, and Intelligent Transport System, focusing on cross-layer protocol 